\title{
The Clock:Cycle complex is a major transcriptional regulator of Drosophila photoreceptors that protects the eye from retinal degeneration and oxidative stress
}

\author{
Juan Jauregui-Lozano $\oplus^{1}$, Hana Hall $\oplus^{1,2}$, Sarah C. Stanhope $\oplus^{1}$, Kimaya Bakhle $\oplus^{1}$, \\ Makayla M. Marlin ${ }^{1}$, Vikki M. Weake $\oplus^{1,2,3 *}$
}

1 Department of Biochemistry, Purdue University, West Lafayette, Indiana, United States of America, 2 Purdue Institute for Integrative Neuroscience, Purdue University, West Lafayette, Indiana, United States of America, 3 Purdue Center for Cancer Research, Purdue University, West Lafayette, Indiana, United States of America

*vweake@purdue.edu

\section{OPEn ACCESS}

Citation: Jauregui-Lozano J, Hall H, Stanhope SC, Bakhle K, Marlin MM, Weake VM (2022) The Clock: Cycle complex is a major transcriptional regulator of Drosophila photoreceptors that protects the eye from retinal degeneration and oxidative stress. PLoS Genet 18(1): e1010021. https://doi.org/ 10.1371/journal.pgen.1010021

Editor: John Ewer, Universidad de Valparaiso, CHILE

Received: September 14, 2021

Accepted: January 8, 2022

Published: January 31, 2022

Peer Review History: PLOS recognizes the benefits of transparency in the peer review process; therefore, we enable the publication of all of the content of peer review and author responses alongside final, published articles. The editorial history of this article is available here: https://doi.org/10.1371/journal.pgen.1010021

Copyright: @ 2022 Jauregui-Lozano et al. This is an open access article distributed under the terms of the Creative Commons Attribution License, which permits unrestricted use, distribution, and reproduction in any medium, provided the original author and source are credited.

Data Availability Statement: All data are fully available without restriction. All the highthroughput data is available at the Gene Expression

\section{Abstract}

The aging eye experiences physiological changes that include decreased visual function and increased risk of retinal degeneration. Although there are transcriptomic signatures in the aging retina that correlate with these physiological changes, the gene regulatory mechanisms that contribute to cellular homeostasis during aging remain to be determined. Here, we integrated ATAC-seq and RNA-seq data to identify 57 transcription factors that showed differential activity in aging Drosophila photoreceptors. These 57 age-regulated transcription factors include two circadian regulators, Clock and Cycle, that showed sustained increased activity during aging. When we disrupted the Clock:Cycle complex by expressing a dominant negative version of Clock ( $\left.\mathrm{Clk}^{\mathrm{DN}}\right)$ in adult photoreceptors, we observed changes in expression of $15-20 \%$ of genes including key components of the phototransduction machinery and many eye-specific transcription factors. Using ATAC-seq, we showed that expression of $\mathrm{Clk}^{\mathrm{DN}}$ in photoreceptors leads to changes in activity of 37 transcription factors and causes a progressive decrease in global levels of chromatin accessibility in photoreceptors. Supporting a key role for Clock-dependent transcription in the eye, expression of $\mathrm{Clk}^{\mathrm{DN}}$ in photoreceptors also induced light-dependent retinal degeneration and increased oxidative stress, independent of light exposure. Together, our data suggests that the circadian regulators Clock and Cycle act as neuroprotective factors in the aging eye by directing gene regulatory networks that maintain expression of the phototransduction machinery and counteract oxidative stress.

\section{Author summary}

Age-associated changes to the retinal transcriptome often correlate with physiological changes, such as loss of visual function and increase in cell death. However, the 
Omnibus (GEO) repository under the specific accession codes outlined below: GSE169328 (D10 ATAC-seq and RNA-seq), GSE184069 (D20 - D60 ATAC-seq), GSE174515 (D20 - D60 and D10 light/ dark RNA-seq), GSE183746 (ClkDN ATAC-seq), GSE184121 (ClkDN RNA-seq). Numerical data underlying graphs are provided in S4 Table and S5 Table.

Funding: This research was funded by the National Eye Institute of the NIH under Award Number R01EY024905 to V.M.W and by the Bird Stair Research Fellowship and Ross Lynn Research Scholar fund (Biochemistry Department, Purdue University) to J. J-L. and by NIH training award 5T32GM125620 to S.C.S. The funders had no role in study design, data collection and analysis, decision to publish, or preparation of the manuscript.

Competing interests: The authors have declared that no competing interests exist. mechanisms that contribute to these transcriptomic changes are poorly understood. Here, we used a genomics/bioinformatics approach to identify transcription factor binding sites with differential activity in aging Drosophila retina outer photoreceptors. Amongst these age-regulated transcription factors, we identify the circadian regulators Clock and Cycle. Using a genetics approach, we find that photoreceptor-specific disruption of the Clock:Cycle complex makes the Drosophila eye susceptible to lightdependent retinal degeneration, and light-independent increase of oxidative stress, showing that a functional circadian clock contributes to visual health and function in Drosophila. Because disruption of circadian rhythms has been associated with the onset of several age-related eye diseases, our data shows that the Drosophila retina serves as a useful model to study how disruption of the circadian clock contributes to neurodegeneration in the retina.

\section{Introduction}

One of the hallmarks of the aging eye, as well as many age-related eye diseases, is the loss of photoreceptor function and survival [1,2]. The aging epigenome and transcriptome of cells in the retina undergo changes that correlate with decreased visual function and increased cell death [3-5]. Importantly, disruption of epigenetic mechanisms is associated with the onset of age-related eye diseases, such as age-related macular degeneration [6,7], suggesting that transcriptional regulation contributes to the changes in homeostasis that are observed in the aging eye. However, we still have only a basic understanding of how the molecular mechanisms that drive the age-associated changes in the transcriptome increase the risk of ocular disease with advanced age.

Transcription factors (TF) function as regulatory hubs of gene expression programs in a highly-tissue specific manner. While several conserved pathways contribute to changes in the aging transcriptome across tissues, such as the longevity-associated FOXO and Insulin axis [810], age-associated changes in gene regulatory networks can be highly specific to individual cell types [5,11]. The emergence of bioinformatic and computational approaches that combine chromatin accessibility data with transcription factor binding sites (TFBS) has allowed researchers to interrogate unbiasedly how transcription factor activity changes at a genomewide scale in different biological conditions by estimating changes in chromatin accessibility around TFBS (see [12] for review on chromatin accessibility data analysis). The majority of chromatin accessibility studies in the eye have focused on development in invertebrate [13] or vertebrate models [14], and TF activity in the developing eye has often been assessed based on transcriptomic-based co-expression inference rather than chromatin accessibility [15]. Further, ATAC-seq analysis of retinal pigmented epithelium from patients with age-related macular degeneration identified global changes in chromatin accessibility at the onset of the disease state, suggesting that differential activity of regulatory elements strongly contributes to the initial stages of age-associated ocular disease [16]. Thus, identification of TFs with differential activity in the aging eye could provide insight into the mechanisms that contribute to the increased risk of retinal degeneration during aging.

Drosophila melanogaster, like humans, experience an age-dependent decline in visual function coupled with increased risk of retinal degeneration [3,17]. Although flies possess a compound eye that differs anatomically from the vertebrate eye, much of the phototransduction machinery is conserved between flies and mammals, with the outer fly photoreceptors resembling vertebrate rods in function $[18,19]$. There are six outer photoreceptors (R1 -R6 cells) 
within each ommatidium that express the light-sensing protein Rhodopsin 1 (Rh1) and are responsible for motion vision and phototaxis, which decline with age [3,17]. Considering its relatively short lifespan, Drosophila provides a useful model system for studying the processes involved in normal aging within specific tissues, such as the eye [20]. To profile the transcriptome and epigenome of specific cell types in the eye, we have developed a cell type-specific nuclei immunoenrichment technique that we have previously used to examine gene expression in aging photoreceptors [3,21,22]. Here, we applied this technique to profile the transcriptome and chromatin accessibility landscapes of Drosophila photoreceptors across an extended time course into relatively old age. By integrating these aging data from photoreceptors, we identified 57 TF motifs that were differentially accessible during aging, suggesting age-dependent changes in TF activity. Amongst these "age-regulated TFs", we identified the binding motif of the master circadian regulators, Clock (Clk) and Cycle (Cyc), which showed sustained increases in activity with age. Using a dominant negative mutant of Clock $\left(\mathrm{Clk}^{\mathrm{DN}}\right)$ that disrupts the Clk:Cyc complex and abolishes rhythmic transcription, we showed that the Clk:Cyc complex has an integral role in controlling gene expression of $15-20 \%$ of active genes, and maintaining global levels of chromatin accessibility in photoreceptors. Further, we show that expression of $\mathrm{Clk}^{\mathrm{DN}}$ in photoreceptors leads to progressive retinal degeneration, which was suppressed when flies were reared in constant darkness. Our data identify a novel neuroprotective role for the circadian clock in the Drosophila eye, and suggest that this role may become increasingly critical in advanced age to prevent retinal degeneration.

\section{Results}

\section{Tissue-specific profiling of the photoreceptor nuclear transcriptome reveals significant changes during early and late aging}

To profile the transcriptome of aging Drosophila photoreceptors, we used our recently improved tissue-specific nuclei immunoenrichment approach [22]. Briefly, we tag nuclei using a Green Fluorescent Protein (GFP) fused to the "Klarsicht, ANC-1, Syne Homology" (KASH) domain, which anchors GFP to the outer nuclear membrane and allows for nuclei purification; $\mathrm{GFP}^{\mathrm{KASH}}$ is expressed in outer photoreceptors under Rh1-Gal4 control, herein referred as Rh1 $>\mathrm{GFP}^{\mathrm{KASH}}$ (Fig 1A). Similar to wild-type strains, such as OregonR and Canton-S [23], $\mathrm{Rh} 1>\mathrm{GFP}^{\mathrm{KASH}}$ flies begin to show a substantial decline in viability after day 60 , with $50 \%$ of male flies dying by day 70 [3]. Thus, to generate a comprehensive aging dataset, we purified photoreceptor nuclei over 10-day windows from day 10 until day 60 and performed RNA-seq using 400 age-matched male flies per biological replicate (Fig 1B).

We assessed the overall variability of the RNA-seq samples using Principal component analysis (PCA). The RNA-seq samples clustered together with $48.3 \%$ of the variation separating the samples by age in a progressive manner (Fig $1 \mathrm{C}$ ). We also observed a similar grouping by age using Pearson's correlation analysis with high concordance between the three biological replicates (Pearson's $r>0.91$ ) (S1 Fig). These observations reveal progressive changes in transcription during aging in photoreceptors, similar to previous studies from our group that aged flies to day 40 (D40) [3]. Differential expression analysis of each time point relative to day 10 (D10), the youngest state, revealed significant changes in gene expression during aging ( $\mathrm{p}$ adj<0.05) (Fig 1D and S1 Table). Hierarchical clustering of the genes that were differentially expressed between any age and D10 revealed that 1412 and 982 genes showed progressive increases, or decreases in expression, respectively. Gene Ontology (GO) term analysis revealed that the genes that were age-upregulated (Clusters 2 and 3) were enriched for several processes including cytoplasmic translation and double strand break repair (Fig 1E). In contrast, the genes that were age-downregulated (Cluster 5) were enriched for neuronal processes, such as 
A

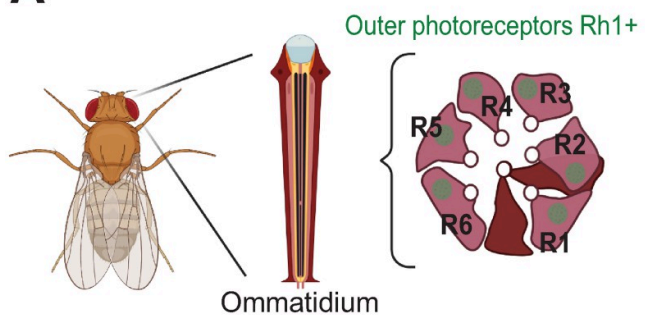

D

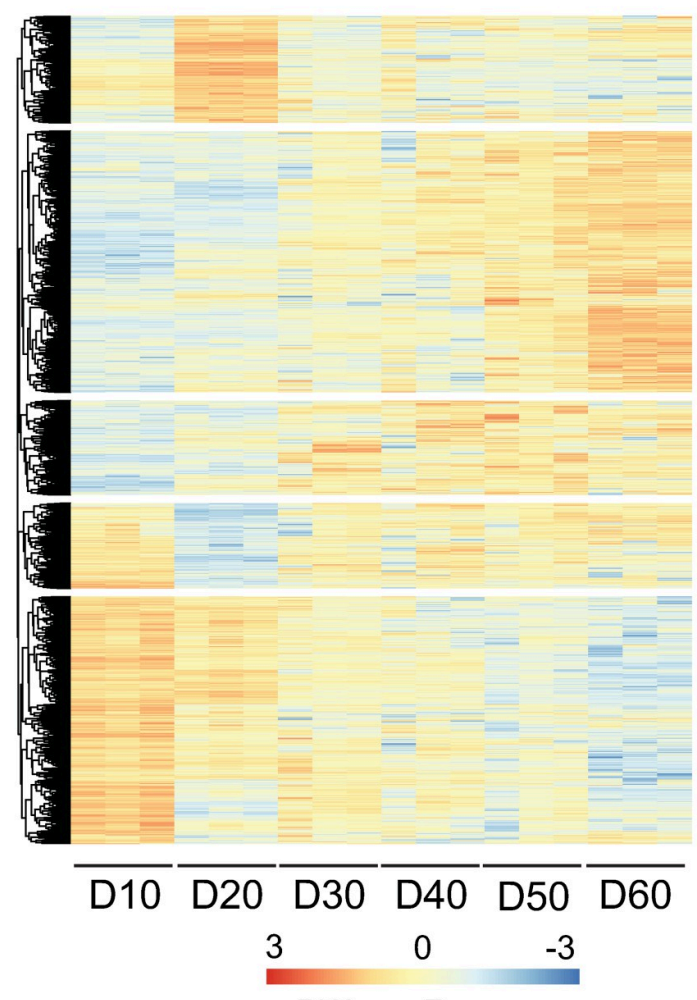

RNA-seq Z-score

B
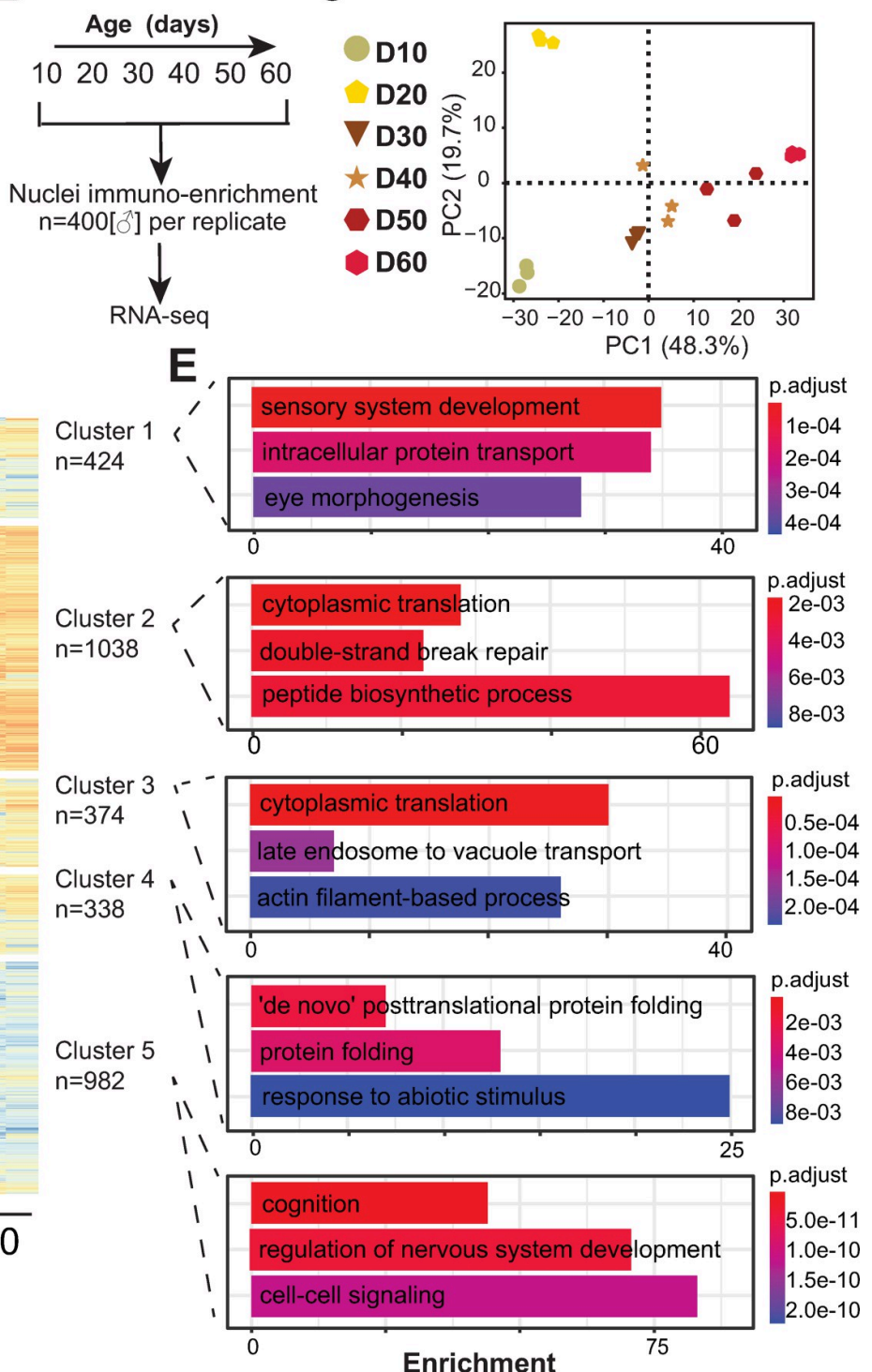

$-04$

$-04$ 政 
are consistent with our previous observations in aging photoreceptors and with aging studies from other tissues $[3,4]$.

\section{Photoreceptors undergo substantial changes in transcription factor activity during aging}

To identify mechanisms that contribute to the age-associated transcriptomic changes in photoreceptors, we sought to evaluate changes in TF activity. TFs regulate transcriptional outputs of their targets by acting as activators or repressors of gene expression at the transcriptional level. Importantly, TF activity can be affected by several factors, including protein levels, localization, and post-translational modifications [24,25]. Because of the technical complexity of isolating intact photoreceptors for proteomic studies, and the relatively low protein abundance of TFs in the eye [26], we used our RNA-seq data to assess how transcript levels of genes associated with the GO category "DNA-binding transcription factor activity, RNA polymerase II-specific" (GO:0000981) changed during aging. Notably, 23\% of TFs showed significant differential expression during aging at the nuclear transcript level (Fig 2A), suggesting that differential TF activity could contribute to the aging transcriptome of photoreceptors. To identify TFs with differential activity during aging, we used $\operatorname{diff} T F$, a software package that estimates genomewide changes in TF motif/binding activity based on differences in aggregate ATAC-seq signal around predicted/validated TF binding sites, or TFBS [27]. We refer to TF motif/binding activity as "TF activity", as defined in the diffTF study [27]. Although diffTF analysis provides an estimate of TF activity, rather than a direct measurement of TF binding to DNA, diffTF was highly ranked as an approach to assay genome-wide changes in TF activity [28]. Thus, we first profiled the chromatin accessibility landscape of aging photoreceptors at the same time-points as for RNA-seq. PCA of ATAC-seq samples revealed that $69.7 \%$ of the variation could be explained by age (Fig 2B), and Pearson correlation analysis showed high concordance between biological replicates. Additionally, accessible peak annotation revealed a stable distribution of peaks through-out aging (S2A Fig). Together with the RNA-seq aging time course, these data indicate that the chromatin accessibility and transcriptional landscape of photoreceptors undergo progressive changes during aging. We then used diffTF to compare all time points relative to D10. The TFBS used for this analysis were generated by CIS-BP, which provides a comprehensive dataset of experimentally validated TFBS [29]. We also examined de novo and known motifs identified using Homer [30] (Fig 2C). We took advantage of the aging RNA-seq time series data to perform diffTF analysis in integrative mode, enabling us to discard TFs that were not expressed in adult photoreceptors. Using this approach, we identified 57 TFs whose binding sites showed significant differential activity during aging (FDR $<0.001)$ (Fig $2 \mathrm{C}$ ), herein referred to as "age-regulated TFBSs". Most of these age-regulated TFs showed continuous changes in activity with age, with nearly two thirds showing increased activity with age. We observed an almost two-fold increase in the number of age-regulated TFs identified at D50 and D60 relative to younger ages, suggesting that late aging is associated with distinct changes in gene regulatory networks (Fig 2D). Hierarchical clustering of these age-regulated TFs by the mean weighted difference between each age comparison resulted in grouping of TFs that are known to associate in vivo, such as Motif 1 Binding Protein (M1BP), DNA replication element factor (Dref) and Boundary element-associated factor of 32kD (BEAF32), which bind topologically associating domains [31]. We refer to TF proteins here using their gene name and symbol (non-italicized), and provide a complete list of all genes/proteins referred to in this study with their corresponding Flybase ID numbers in S3 Table. We also identified Moira (mor), which physically interacts with Similar (sima) [32]. Additionally, we identified Seven up (svp) and PvuII-PstI homology 13 (Pph13), which physically interact to regulate eye-specific 
A

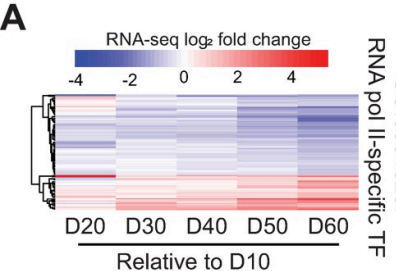

$\mathbf{E}$

B

ATAC-seq

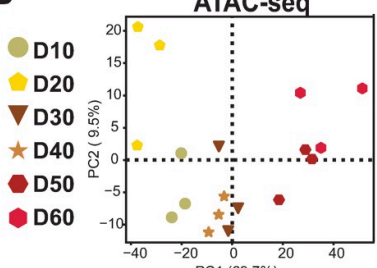

C

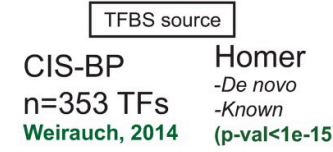

Weirauch, 2014

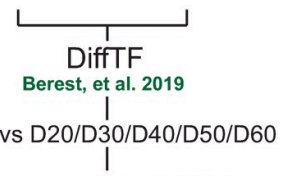

Age-regulated TFBS

D
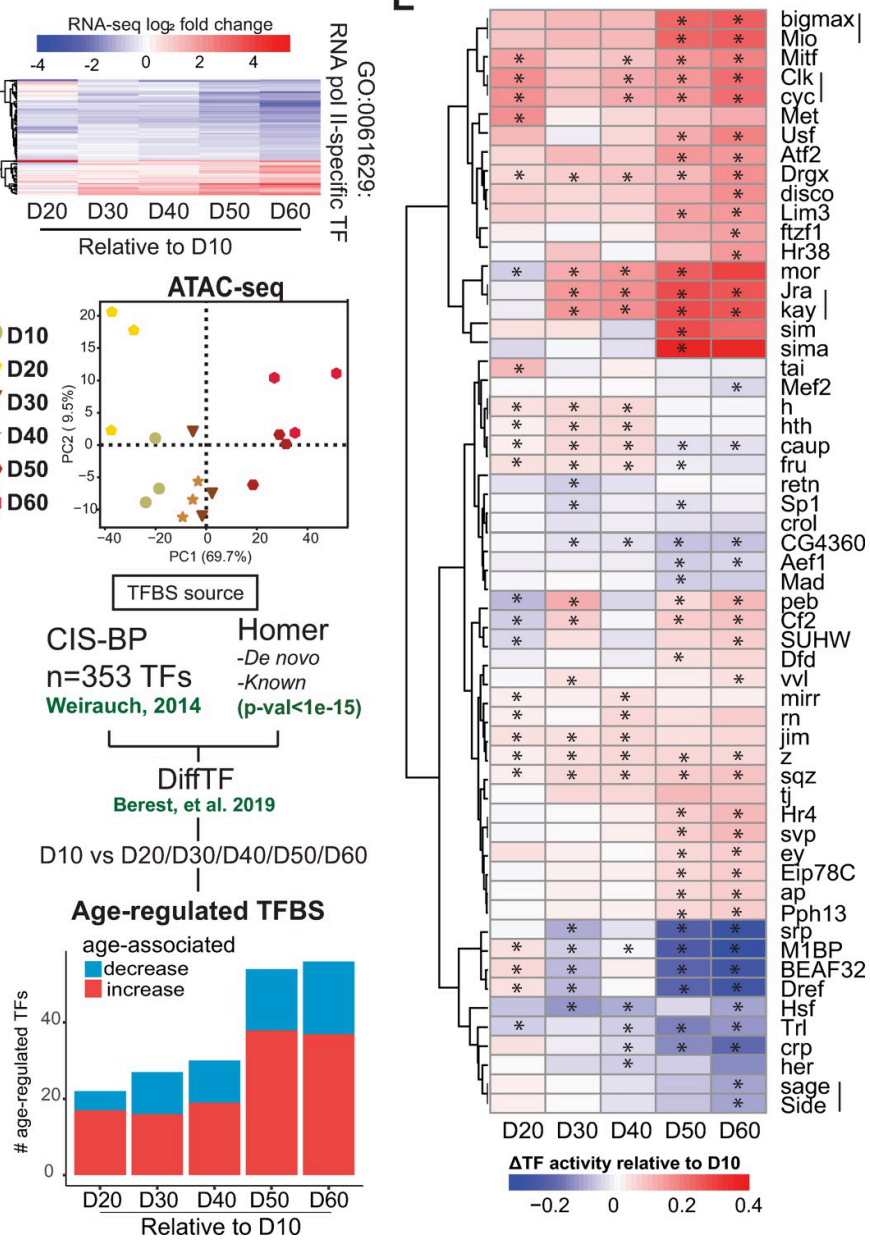

Fig 2. Photoreceptors undergo substantial changes in TF activity during aging. A. Heatmap of RNA-seq fold change during aging for significantly differentially expressed genes (DEGs; $\mathrm{p}$-adj $<0.05,|\mathrm{FC}|>1.5$ relative to D10) associated with the GO term "RNA polymerase II-specific DNA-binding transcription factor binding" (GO:0061629). Genes are hierarchically clustered by $\log _{2}$ fold change values. B. PCA of aging ATAC-seq samples based on the read distribution over binned genome. C. Schematic of differential transcription factor (TF) analysis approach using diffTF. D. Bar plot indicating the number of significant age-regulated TFs at each age relative to D10 (p-adj $<0.001)$. E. Hierarchical clustered heatmap of age-regulated TFs with significant changes in activity between any age and D10 (asterisk, p-adj $<0.01$ ). Scale represents the relative change in TF activity with red showing higher TF activity in old samples relative to D10, and blue indicating an age-associated decrease in activity. TFs that bind a common motif and cluster together are indicated by a vertical line.

https://doi.org/10.1371/journal.pgen.1010021.g002

transcriptional programs together with Eyeless (ey) [33]. Considering that svp, ey, and Pph13 show modest but significant increases in TF activity with age, our data also indicate that photoreceptor identity remains distinct even at advanced age in flies.

The stress response is transcriptionally regulated during aging across a broad range of tissues [34]. Accordingly, we identified several TFs that are involved in the regulation of stress response genes as having differential activity with age including the Drosophila JUN-FOS complex that is formed by Jun-related antigen (Jra) and Kayak (kay) [35], the Drosophila homolog of Hypoxia response factor HIF-1a, sima [36], Heat shock factor (Hsf) [37], and Activating transcription factor-2 (Atf-2) [38,39]. We also identified Cropped (crp), which has previously been associated with aging through in silico analysis due to interactions with DNA repair pathways [40]. 
One of the most interesting changes in TF activity during aging was in the activity of the master circadian regulators Clock $(\mathrm{Clk})$ and $\mathrm{Cycle}(\mathrm{Cyc})$, which showed a progressive increase in TF activity during aging. Clk and Cyc bind the same CACGTG motif upon heterodimerization, explaining the identification and clustering of both factors in the diffTF analysis, which uses DNA sequence motifs as the TFBS source (S2B Fig). The circadian clock is a molecular time keeper that controls rhythmic behaviors, such as locomotor behavior, which is coordinated by pacemaker neurons located in the brain [41]. In Drosophila, many peripheral tissues also contain working clocks, including the fat body, Malpighian tubule cells, and the retina [42]. Importantly, aging has been associated with changes in the circadian clock $[43,44]$. We note that flies for the aging time course experiment were raised under a 12:12 hour light-dark cycle, and were harvested at relative Zeitgeber time (ZT) $6+/-1$ hour (see Materials and Methods), suggesting that the enrichment of Clk and Cyc in the diffTF analysis is not simply due to a time-of-day bias in sample collection. Taken together, our data identify a subset of TFs that exhibit significant changes in activity during aging in photoreceptors, including TFs associated with stress response and circadian rhythm.

\section{Clock regulates the transcriptional output of phototransduction genes in photoreceptors}

Clk and Cyc are basic Helix-Loop-Helix (bHLH)-TFs that form a heterodimer (Clk:Cyc) to control rhythmic expression of their targets. Canonical transcriptional targets of the Clk:Cyc complex include core clock genes, such as vrille (vri), PAR-domain protein 1 (Pdp1), timeless (tim), period (per), and clockwork orange (cwo). However, Clk and Cyc also regulate transcription of many other genes either directly or indirectly, and these genes are often referred as "Clock-output genes", and can include tissue-specific genes $[45,46]$. To evaluate the biological role of Clock-dependent transcription specifically in photoreceptors, we disrupted the Clk:Cyc complex by expressing a dominant negative version of Clk $\left(\mathrm{Clk}^{\mathrm{DN}}\right)$ specifically in outer photoreceptors under Rh1-Gal4 control. Clk ${ }^{\mathrm{DN}}$ lacks the basic DNA binding domain, impairing its recruitment to DNA without disrupting its ability to form a heterodimer with Cyc (Fig 3A) [47]. Expression of $\mathrm{Clk}^{\mathrm{DN}}$ inhibits Clock-dependent transcription and rhythmic behaviors in vivo when expressed in pacemaker or antennal neurons [47]. To facilitate nuclei immunoenrichment for RNA-seq analysis, we co-expressed UAS-GFP ${ }^{\mathrm{KASH}}$. As a control, we profiled the transcriptome of photoreceptors that expressed LacZ, herein referred as Rh1 $>\mathrm{Ctrl}$. We collected both $\mathrm{Rh} 1>\mathrm{Clk}^{\mathrm{DN}}$ and $\mathrm{Rh} 1>\mathrm{Ctrl}$ flies at ZT 9, when Clock-dependent transcription is active $[48,49]$, harvesting flies at D1 and D10 to study the progressive effect of disrupting the circadian clock in adult photoreceptors (Fig 3B). We note that Rh1-Gal4 activity begins in the very late stages of pupal development [50]; thus $\mathrm{Rh} 1>\mathrm{Clk}^{\mathrm{DN}}$ flies have a disrupted Clk:Cyc complex in adult photoreceptors, but not in the developing eye.

Differential gene expression (DGE) analysis of $\mathrm{Rh} 1>\mathrm{Clk}^{\mathrm{DN}}$ relative to $\mathrm{Rh} 1>\mathrm{Ctrl}$ at either D1 or D10 revealed a consistent downregulation of direct Clk:Cyc targets such as vri, per, tim and $P d p 1$ (Figs $3 \mathrm{C}$ and S2A and S2 Table). We also observed a significant upregulation of Clk itself, which we showed by qPCR reflects expression of $C l k^{D N}$ rather than the endogenous wild-type Clk (S2B Fig). When we compared the control D1 and D10 flies, we did not observe differential expression of core clock genes (i.e. vri, tim, per), suggesting there is little change in the circadian clock in the early stages of adult life. In addition, only 147 genes changed in control flies between D1 and D10, indicating there is relatively little change in gene expression in general at these early stages of adult life in photoreceptors. In contrast, expression of Clk ${ }^{\mathrm{DN}}$ led to significant changes in expression of $15 \%$ and $22 \%$ of actively transcribed genes in photoreceptor at D1 and D10, respectively. These data demonstrate that continued expression of 
A

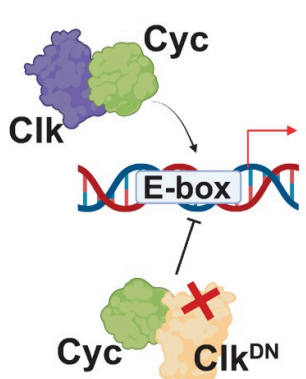

B

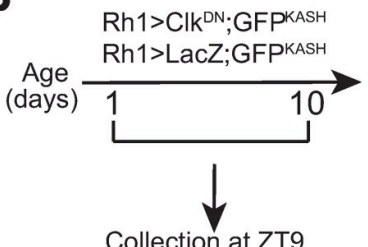

Nuclei immuno-enrichment

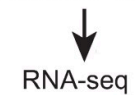

D

E (CBGs)

\section{G}

Phototransduction

ninaE, trp, linc, inaF-B, $r d g B, A r r 2, r d g C, P I P 82$, Arr1, trpl, ninaC, dnc, Calx, slgA, Itpr, Gbeta76C Circadian rhythm cwo, vri, Moe, tim, per, Clk, homer, nvy, Ih Transcription factors psq, Lim1, Dif, oc, ey, Xrp1, ttk, shn, ab, vnd, Glu4EF, dwg, Mnt, CrebA, fuss

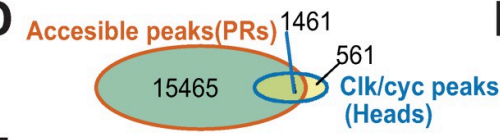

(Heads)
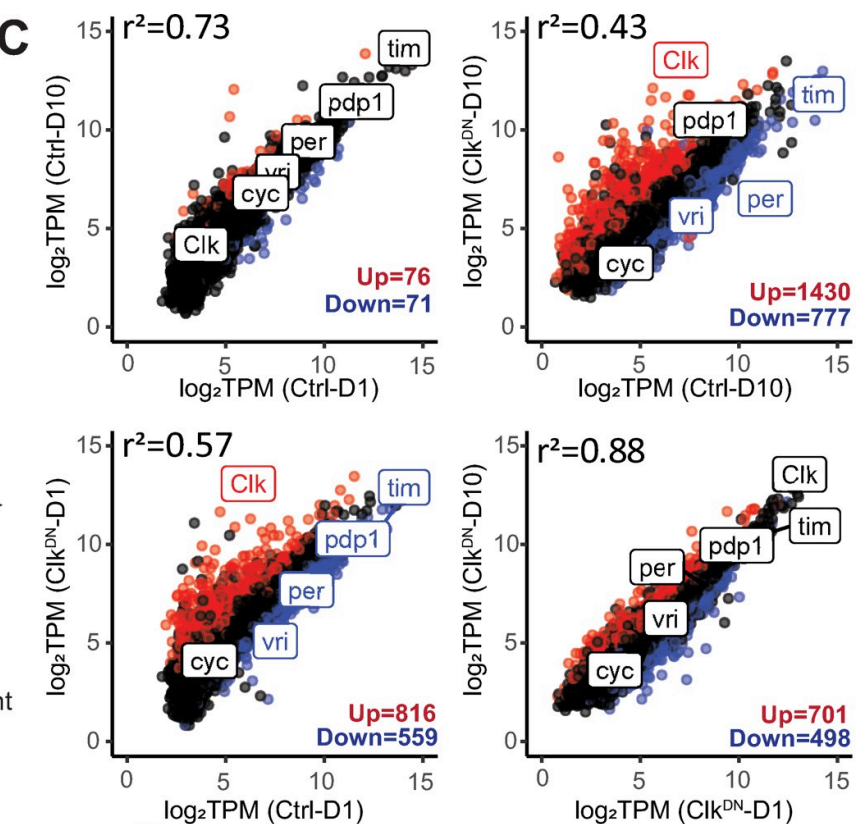

$\mathbf{F}$

(

protein serine/threonine kinase activity

neuron projection development

DNA-binding transcription factor activity

RNA polymerase II-specific eye development

H motor activity

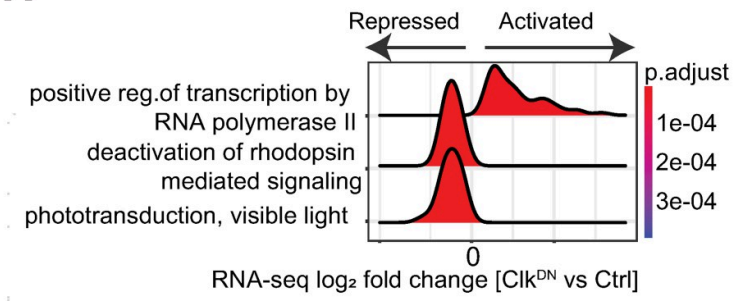

Fig 3. Expression of $\mathrm{Clk}^{\mathrm{DN}}$ in photoreceptors leads to genome-wide changes in TF activity and a widespread decrease in chromatin accessibility. A. Schematic of Clk:Cyc activity. Expression of $\mathrm{Clk}^{\mathrm{DN}}$ disrupts Clock-dependent transcription because it binds Cyc but does not contain the DNA binding domain. B. Schematic for the RNA-seq experiments. Flies that express the $\mathrm{GFP}^{\mathrm{KASH}}$ tag and either $\mathrm{Clk}^{\mathrm{DN}}$ or LacZ (control) in photoreceptors were aged to D1 or D10 prior to NIE. Flies were harvested at the indicated ZT. C. Scatter plot of mean expression (TPM, $n=3)$ for the indicated pair-wise comparisons. Up- and down-regulated DEGs (p-adj<0.05, $|\mathrm{FC}|>1.5$ ) are colored in red and blue, respectively. Core clock genes $C l k, c y c, v r i, p e r, P d p 1$ and tim are highlighted. D. Genomic overlap of accessible peaks in photoreceptors and Clk:Cyc binding sites identified using ChIP-seq in [51]. E. Venn diagram comparing overlap of Clock-bound genes (CBGs-green) with genes that were differentially expressed (adj $\mathrm{p}<0.05,|\mathrm{FC}|>1.5$ ) in $\mathrm{Rh} 1>\mathrm{Clk}^{\mathrm{DN}}$ relative to control. F. GO term analysis of Clock direct and indirect targets. G. Table showing selected genes identified as Clock direct target genes that were associated with the indicated biological processes. H. Ridge plot of selected GO terms in flies expressing $\mathrm{Clk}^{\mathrm{DN}}$ versus control at D1 identified using Gene Set Enrichment Analysis.

https://doi.org/10.1371/journal.pgen.1010021.g003

$\mathrm{Clk}^{\mathrm{DN}}$ leads to progressive dysregulation of gene expression in photoreceptors at nearly a quarter of expressed genes.

To further identify direct versus indirect targets of the Clk:Cyc complex, we compared previously published high-confidence binding sites identified for Clk and Cyc using ChIP-seq in 
Drosophila heads and bodies [51] with our list of accessible peaks obtained with ATAC-seq data from D10 photoreceptors. Genomic overlap analysis revealed that $10 \%$ of accessible peaks contained an experimentally identified Clk:Cyc TFBS (Fig 3D). Next, we annotated these photoreceptor-specific TFBS $(\mathrm{n}=1461)$ to their nearest transcription start site (TSS) and found that 1004 photoreceptor-expressed genes contained a potential Clk:Cyc binding site. We refer to these 1004 genes as "Clock-bound genes" or CBGs (Fig 3E), which we compared to genes that were differentially expressed in $\mathrm{Rh} 1>\mathrm{Clk}^{\mathrm{DN}}$ relative to $\mathrm{Rh} 1>\mathrm{Ctrl}$ at either $\mathrm{D} 1$ or D10. We reasoned that if a gene is bound by Clk:Cyc and is differentially expressed, then it can be classified as a "direct target" of Clk:Cyc. Using these criteria, we identified 475 direct Clk:Cyc targets in photoreceptors. In contrast, we identified a far greater number of genes (2253) that are likely to be indirect targets of Clk:Cyc regulation, at least based on the available ChIP-seq data from whole heads [51]. Consistent with the predicted role of Clk:Cyc as transcriptional activators, Clk:Cyc direct targets were more likely to be downregulated relative to indirect targets upon expression of $\mathrm{Clk}^{\mathrm{DN}}$ (S3C Fig). GO term analysis of direct Clk:Cyc targets showed significant enrichment of genes associated with several biological processes, including phototransduction and circadian rhythm (Fig 3F). However, both direct and indirect Clk:Cyc targets were enriched for processes including TF activity and eye development. This is consistent with previous reports that have proposed that the Clk:Cyc complex acts at the top of a TF hierarchy, directly regulating transcript levels of key TFs in specific cell types, thereby indirectly regulating expression of their target genes [51,52]. Notably, individual inspection of the TFs that were classified as direct Clk:Cyc targets confirmed that several eye-specific TFs such as ocelli (oc) otd) and eyeless (ey) are bound at their promoters directly by Clk and Cyc (Fig 3G). To further identify the magnitude of changes in expression of these pathways upon disruption of Clk:Cyc, we performed gene set enrichment analysis comparing $\mathrm{Clk}^{\mathrm{DN}}$ vs Control at either D1 or D10 and obtained ridge plots showing the density of fold change for the genes associated with each pathway. Whereas upregulated genes showed significant enrichment of several biological processes, including TF activity, downregulated genes were associated with several phototransduction-associated pathways, including deactivation of rhodopsin signaling (Figs $3 \mathrm{H}, \mathrm{S} 3 \mathrm{D}$ and S3E).

Because light entrains the circadian clock to activate Clock-mediated transcription, we next performed RNA-seq in photoreceptors from $\mathrm{Rh} 1>\mathrm{GFP}^{\mathrm{KASH}}$ flies reared in constant darkness (DD). Under these conditions, we observed differential expression of genes associated with several biological processes, including phototransduction and circadian rhythm in dark raised flies relative to LD (S3F and S3G Fig). Because these processes were also enriched in the genes with differential expression upon disruption of Clk:Cyc activity, our data suggest that these biological processes are normally regulated by the circadian clock in photoreceptors in response to the light/dark cycle.

Taken together, these data show that the Clk:Cyc complex is a major transcriptional regulator of the photoreceptor transcriptome, including the phototransduction pathway. Additionally, our data suggest that Clk activity regulates gene regulatory networks by regulating expression of TFs that in turn direct expression of a large proportion of the transcriptome in photoreceptors.

\section{Expression of $\mathrm{Clk}^{\mathrm{DN}}$ leads to genome-wide changes in TF activity and a widespread decrease in chromatin accessibility}

Because expression of $\mathrm{Clk}^{\mathrm{DN}}$ led to transcriptional dysregulation of TFs, we further investigated the TFs that showed changes in transcript level upon disruption of the Clk:Cyc complex. Gene Concept Network (cnet) plots demonstrated significant enrichment of 62 TFs amongst 


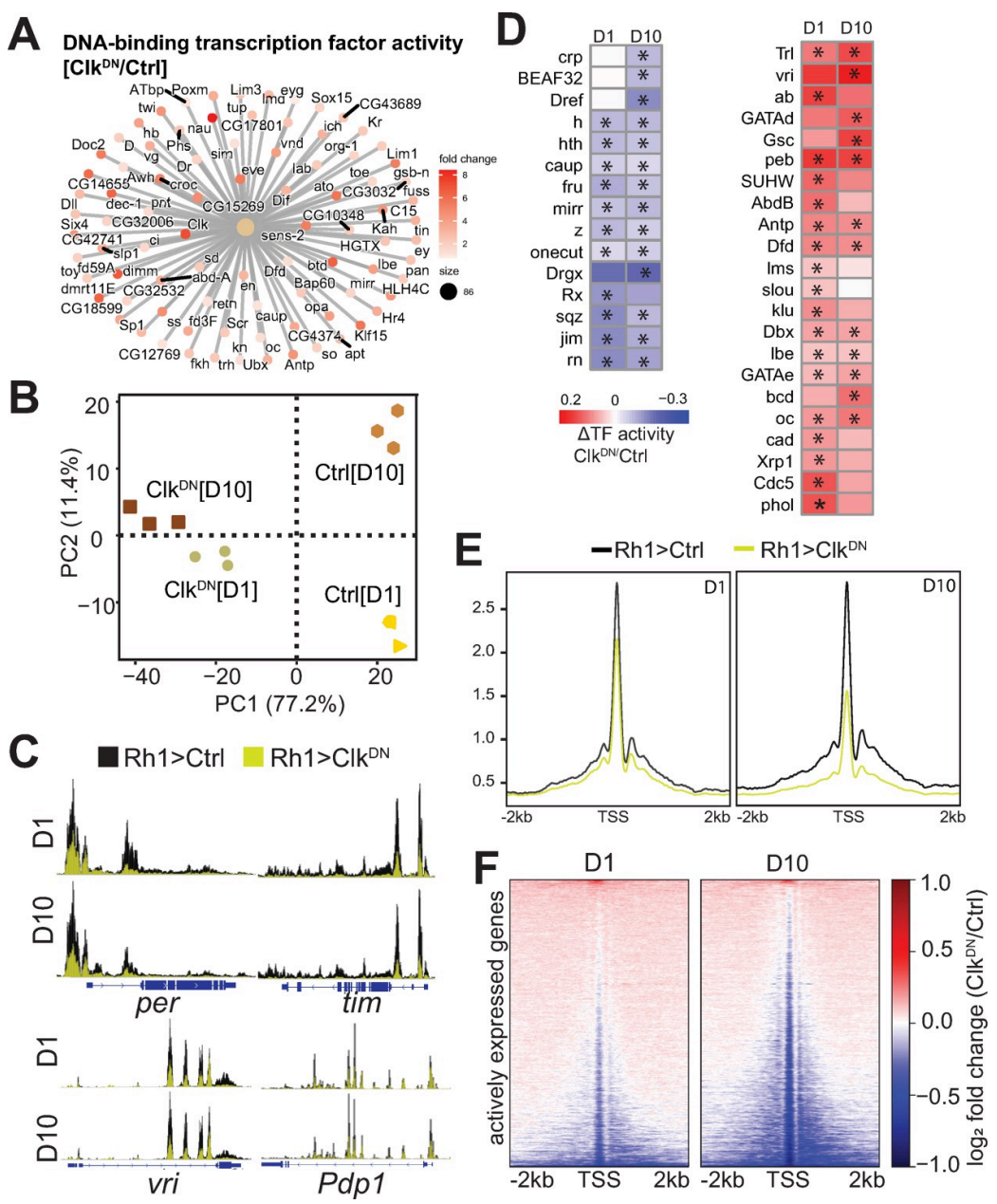

Fig 4. Clock activity promotes changes in TF activity and maintains global levels of chromatin accessibility in photoreceptors. A. Cnet plot of genes associated with the GO term "DNA-binding transcription factor activity". Color represents change in expression in $\mathrm{Clk}^{\mathrm{DN}}$ relative to control at D1. B. PCA of Clk ${ }^{\mathrm{DN}}$ and control ATAC-seq samples based on the read distribution over a 10-kb binned genome. C. Genome browser inspection of CPM-normalized ATAC-seq signal for selected core Clock genes (per, tim, vri and Pdp1). Scale is normalized to the same height in each comparison. ATAC-seq from Ctrl flies is labeled in black, and $\mathrm{Clk}^{\mathrm{DN}}$ in yellow. D. Hierarchical clustered heatmap of significant Clock-regulated transcription factors identified between $\mathrm{Rh} 1>\mathrm{Clk}{ }^{\mathrm{DN}}$ and $\mathrm{Rh} 1>\mathrm{Ctrl}$ at either D1 or D10. Scale represents the relative change in TF activity. E. CPM-normalized gene metaplots of ATAC-seq signal centered around Transcription Start Sites (TSS). F. ATAC-seq fold change heatmaps of signal centered around TSS for all actively expressed genes in photoreceptors. Fold change is obtained by dividing signal from $\mathrm{Rh} 1>\mathrm{Clk}^{\mathrm{DN}}$ relative to Rh1 $>$ Ctrl, and $\log ^{2}$ transformed to center changes around zero (no change).

https://doi.org/10.1371/journal.pgen.1010021.g004

the upregulated genes in flies expressing $\mathrm{Clk}^{\mathrm{DN}}$ (Fig 4A). Based on these changes in TF expression, we wondered if expression of $\mathrm{Clk}^{\mathrm{DN}}$ would also lead to significant changes in TF activity relative to Rh1 $>$ Ctrl flies. To test this, we profiled chromatin accessibility in $\mathrm{Rh} 1>\mathrm{Clk}{ }^{\mathrm{DN}}$ versus control, and integrated the RNA-seq and ATAC-seq data using the diffTF approach, as in Fig 2. PCA analysis of the accessible chromatin landscape revealed that $77 \%$ of the variation was explained by expression of $\mathrm{Clk}^{\mathrm{DN}}$ (Fig 4B). In addition, Clk:Cyc targets, such as tim, per, $v r i$ and $P d p 1$, exhibited significant decreases in accessibility through-out their gene bodies upon expression of $\mathrm{Clk}^{\mathrm{DN}}$ (Fig 4C). Importantly, genomic annotation of accessible peaks revealed a stable distribution of peaks in all samples (S4A Fig), suggesting that disruption of the Clk:Cyc complex does not lead to overall changes in the genome-wide distribution of 
accessible peaks. Rather, these data show that the Clk:Cyc complex promotes chromatin accessibility at target genes, consistent with the well characterized role of Clk:Cyc in transcription activation [53].

Using diffTF, we identified $37 \mathrm{TFs}$ with differential activity upon expression of $\mathrm{Clk}^{\mathrm{DN}}$, or "Clock-regulated TFs". Whereas 15 TFs had decreased activity, 22 showed increased activity in $\mathrm{Rh} 1>\mathrm{Clk}^{\mathrm{DN}}$ relative to Rh1 $>\mathrm{Ctrl}$ (Fig 4D). Interestingly, several of the genes encoding these Clock-regulated TFs are also directly bound by Clk and Cyc at their promoters (see Fig 3), including the eye-specific TFs oc/Otd and ey, as well as Xrp1, onecut, crp, and Trl. We also identified increased TF activity of the circadian regulator $v r i$, which represses transcription of $C l k$ [54]. Consistent with the reported role of $v r i$ as a repressor, we observed decreased levels of wild-type $C l k$ transcript in $\mathrm{Rh} 1>\mathrm{Clk}^{\mathrm{DN}}$ flies using qPCR with primers that differentiate between the wild-type and dominant negative version (see S2B Fig). Thus, these data suggest that the Clk:Cyc complex contributes to the maintenance of gene regulatory networks in photoreceptors by regulating transcript levels and/or TF motif/binding activity of many transcription factors.

Visual inspection of chromatin accessibility tracks showed that the $\mathrm{Rh} 1>\mathrm{Clk}^{\mathrm{DN}}$ flies had an overall decrease in chromatin accessibility around promoters and throughout gene bodies (Fig 4C). To further evaluate this, we obtained gene metaplots averaging the ATAC-seq signal around promoters for all actively expressed genes in $\mathrm{Rh} 1>\mathrm{Clk}^{\mathrm{DN}}$ and $\mathrm{Rh} 1>\mathrm{Ctrl}$ flies. Strikingly, we observed that expression of $\mathrm{Clk}^{\mathrm{DN}}$ led to a widespread decrease in chromatin accessibility around transcription start sites, and this decrease in global accessibility was exacerbated at D10 relative to D1 (Fig 4E). These gene-based observations were corroborated by peakbased quantification (S4B Fig). Thus, our data suggest that disruption of the Clk:Cyc complex results in a global decrease in chromatin accessibility in photoreceptors, even though Clk and Cyc only have 475 direct gene targets in this cell type (Fig 3E). To further gain insight if these changes in accessibility were progressive between D1 and D10 for a given gene, we obtained heatmaps of ATAC-seq fold change signal for actively expressed genes at either D1 or D10 in which both heatmaps were sorted identically (Fig 4F). These heatmaps revealed that genes that had decreased accessibility at D1 also showed sustained decreases in accessibility at D10, suggesting that disruption of the Clk:Cyc complex has a role in promoting chromatin accessibility in photoreceptors at a large fraction of actively expressed genes, although our data does not identify if this role is direct or indirect.

Taken together, our data shows that expression of $\mathrm{Clk}^{\mathrm{DN}}$ leads to dysregulation of TF levels and/or activity, suggesting the Clk:Cyc modulates gene regulatory networks associated with these TFs. In addition, expression of $\mathrm{Clk}^{\mathrm{DN}}$ leads to a widespread decrease in chromatin accessibility that is independent from changes in gene expression, suggesting that the circadian clock contributes to the global maintenance of chromatin remodeling of actively expressed genes in Drosophila photoreceptors.

\section{Disruption of Clock activity leads to light-dependent retinal degeneration and light-independent accumulation of oxidative stress}

Disruption of the phototransduction machinery by mutations in phototransduction genes that leads to loss of function or decreased expression is often associated with retinal degeneration [55]. Consistent with the GSEA analysis (see Fig 3H), cnet plots evaluating RNA-seq fold change for genes associated with phototransduction revealed significant decreases in transcript levels of 22 genes in flies expressing $\mathrm{Clk}^{\mathrm{DN}}$ (Figs 5A and S5). These genes included many phototransduction components whose loss leads to light-dependent retinal degeneration, such as $C d s, A r r 2, r d g B, \operatorname{trp}, r d g C$, and ninaC (marked with a red asterisk, Fig 5A) [55]. Thus, we 
A

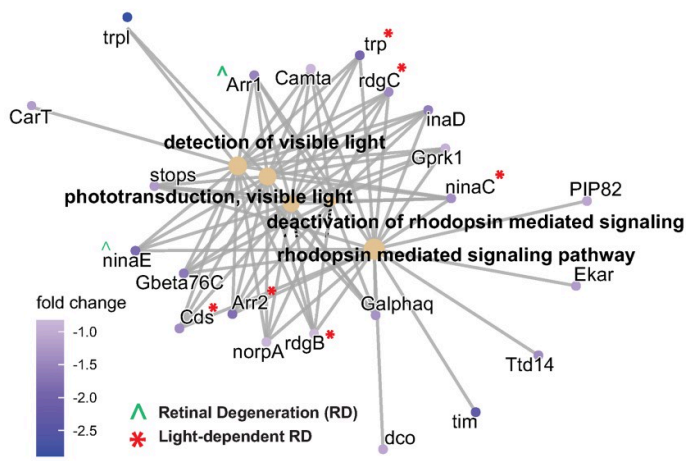

C

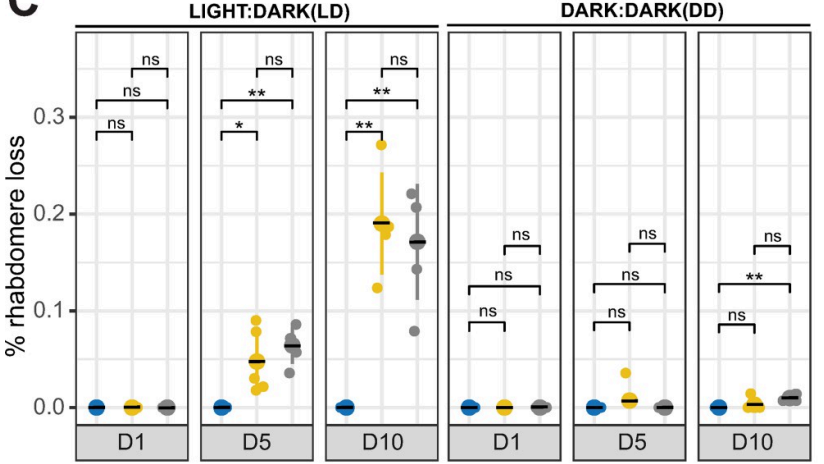

$\mathrm{Rh} 1>\phi \mathrm{Ctrl} \phi \mathrm{Clk}^{\mathrm{DN}}[\mathrm{chr} 2] \phi \mathrm{Clk}^{\mathrm{DN}}[\mathrm{chr} 3]$

B

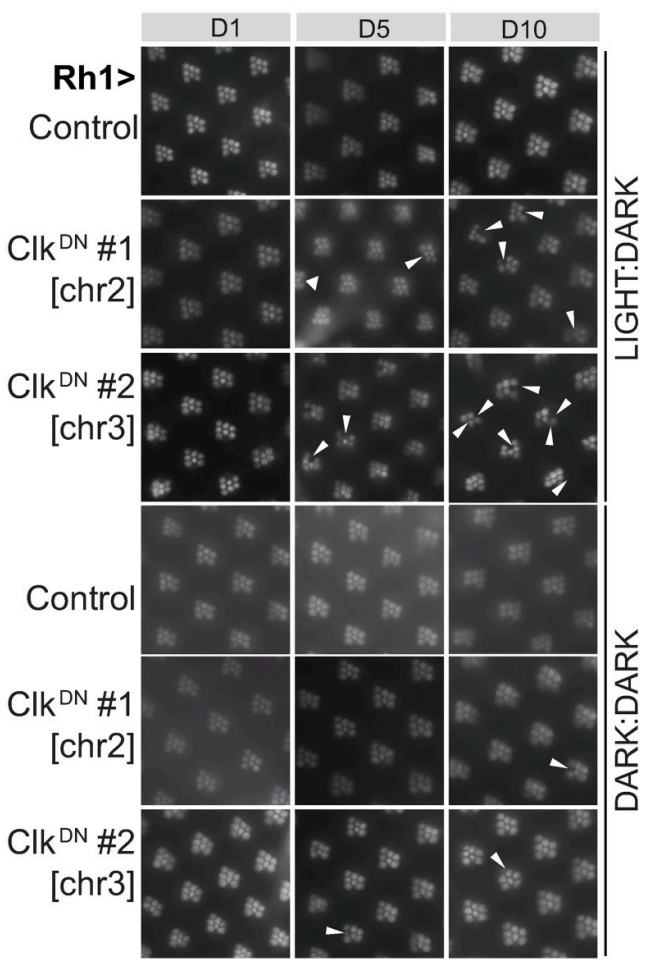

GO Biological Pathways
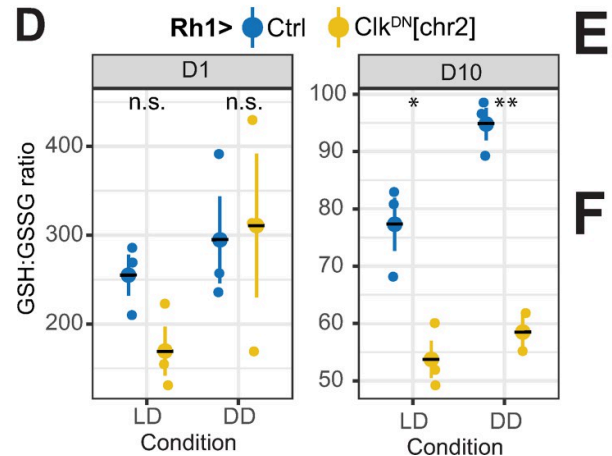

E

response to unfolded protein chaperone cofactor-dependent protein refolding cellular response to topologically incorrect protein chaperone-mediated protein folding

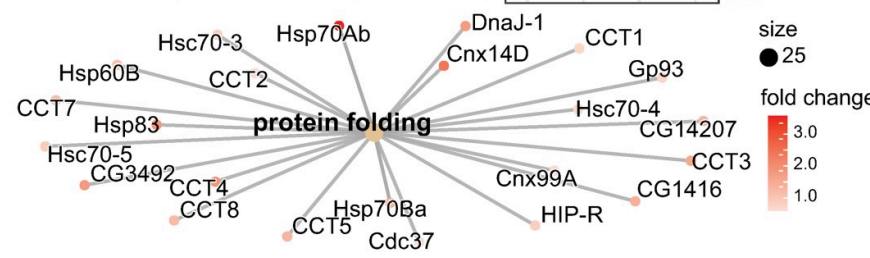

Fig 5. Expression of $\mathrm{Clk}^{\mathrm{DN}}$ leads to light-dependent retinal degeneration and light-independent accumulation of oxidative stress. A. Cnet plot of DEGs (p-adj $<0.05,|\mathrm{FC}|>1.5$ ) in $\mathrm{Rh} 1>\mathrm{Clk}^{\mathrm{DN}}$ relative to $\mathrm{Rh} 1>\mathrm{Ctrl}$ at $\mathrm{D} 1$, associated with phototransduction-related GO terms. Genes associated with light-dependent and -independent retinal degeneration based on published literature are indicated by accent or asterisks. B. Representative images of eyes from flies expressing $\mathrm{Clk}^{\mathrm{DN}}$ versus control at the indicated age reared in light:dark (LD-top) or constant dark (DD—bottom) conditions. Images were obtained using optical neutralization (see Materials and Methods). Arrows indicate position of missing/degenerated rhabdomeres. C. Scatter plot showing quantification of retinal degeneration in panel $\mathrm{B}$. Individual eyes are shown as circles with means shown as horizontal black lines overlaying large circle $(n=5)$. The distribution for each LD reared sample was compared with the DD control for the same age using ANOVA followed by Tukey-HSD comparing ages, samples, and condition (L:D vs D:D). "n.s." = not significant, "**" = FDR <0.05, “**” = FDR <0.005). D. Reduced(GSH):Oxidized(GSSG) Glutathione ratios in dissected eyes from flies of the indicated genotype, age, and condition. Statistics were performed as in panel C. E. Bar plots representing enriched GO terms amongst the genes that were upregulated in $\mathrm{Rh} 1>\mathrm{Clk}^{\mathrm{DN}}$ at D10 relative to D1. F. Cnet plots of genes identified in panel $\mathrm{E}$.

\section{https://doi.org/10.1371/journal.pgen.1010021.g005}

hypothesized that expression of $\mathrm{Clk}^{\mathrm{DN}}$ in Drosophila photoreceptors would lead to retinal degeneration. To test this, we examined photoreceptor integrity using optical neutralization (see Materials and Methods) in Rh1 $>\mathrm{Clk}^{\mathrm{DN}}$ and $\mathrm{Rh} 1>\mathrm{Ctrl}$ flies that were maintained in either standard 12:12 hour light:dark (LD) or free-running, constant darkness (DD) conditions. We 
note that all of the flies used in this study had pigmented eyes (S5B Fig), and were not susceptible to light-mediated damage as is the case for $w^{1118}$ flies. We found that $\mathrm{Rh} 1>\mathrm{Clk}^{\mathrm{DN}}$ flies presented progressive retinal degeneration starting at day 5 , when raised under standard light: dark (LD) conditions relative to Rh1>Ctrl flies (Fig 5B top and 5C left). Importantly, photoreceptors were intact at earlier adult stages just after eclosion (D1), although qPCR analysis from 1-day old heads shows that the $C l k^{D N}$ transcript is already expressed at this age (S3B Fig). We observed retinal degeneration in two independent $U A S-C l k^{D N}$ lines inserted on different chromosomes, suggesting that retinal degeneration is unlikely to result from insertion position of the transgene; moreover, all experiments were performed in progeny hemizygous for the $U A S-C l k^{D N}$ transgene. To test if the retinal degeneration resulting from $\mathrm{Clk}^{\mathrm{DN}}$ expression was dependent on light exposure, we raised $\mathrm{Rh} 1>\mathrm{Ctrl}$ and $\mathrm{Rh} 1>\mathrm{Clk}^{\mathrm{DN}}$ flies in constant darkness (DD) and monitored retinal degeneration. Strikingly, rearing $\mathrm{Rh} 1>\mathrm{Clk}^{\mathrm{DN}}$ flies in constant darkness prevented the onset of retinal degeneration in both $\mathrm{Clk}^{\mathrm{DN}}$ lines (Fig 5B-bottom and $5 \mathrm{C}$-right) demonstrating that expression of $\mathrm{Clk}^{\mathrm{DN}}$ results in light-dependent retinal degeneration in adult Drosophila photoreceptors. Since $\mathrm{Clk}^{\mathrm{DN}}$ expression resulted in light-dependent retinal degeneration, we next asked if expression of the analogous dominant negative for its partner Cycle $\left(\mathrm{Cyc}^{\mathrm{DN}}\right)$ caused a similar phenotype. Surprisingly, we did not observe any retinal degeneration in Rh1 $>$ Cyc ${ }^{\mathrm{DN}}$ flies reared in standard LD conditions at either D5 or D10 (S5C Fig). When we examined the relative level of $C l k$ and $c y c$ transcripts in photoreceptors, we found that Clk transcripts are 10-times more abundant than $c y c$ (S5D Fig). In addition, proteomic analysis of fly heads during the day revealed that there are about 5-fold fewer peptides corresponding to Cyc versus Clk [48]. Thus, the differences in phenotype between $\mathrm{Clk}^{\mathrm{DN}}$ and $\mathrm{Cyc}^{\mathrm{DN}}$ flies could arise because of differences in protein abundance; however, it remains possible that Clk and/or Cyc have independent functions in photoreceptors outside of the canonical Clk:Cyc complex.

The circadian clock has also been shown to be necessary to protect cells against oxidative stress. For example, flies carrying the $\mathrm{Clk}^{J r k}$ allele, which produces a mis-spliced $\mathrm{Clk}$ transcript and leads to decreased clock activity [56], show increased levels of reactive oxygen species (ROS) in aging brains [57]. Since light is a major source of oxidative stress in the eye [58] and $\mathrm{Rh} 1>\mathrm{Clk}^{\mathrm{DN}}$ showed light-dependent retinal degeneration, we hypothesized that $\mathrm{Rh} 1>\mathrm{Clk}^{\mathrm{DN}}$ flies exposed to light had increased oxidative stress levels relative to Rh1 $>$ Ctrl flies or flies reared in DD conditions. To test this, we performed a targeted metabolite assay to measure the ratio of reduced and oxidized glutathione (GSH:GSSG) in $\mathrm{Rh} 1>\mathrm{Clk}^{\mathrm{DN}}$ and Rh1 $>\mathrm{Ctrl}$ flies reared in LD or DD conditions at D1 and D10. In this assay, a lower GSH:GSSG ratio is indicative of increased oxidative stress levels [59]. We note that given the technical limitations for isolation of intact photoreceptors, we performed these targeted metabolite assays using dissected eyes. Using this approach, we found that oxidative stress levels did not show significant changes at D1 in any condition (Fig 5D-left). Unexpectedly, we observed a significant decrease in the GSH:GSSG ratio in $\mathrm{Rh} 1>\mathrm{Clk}^{\mathrm{DN}}$ flies relative to $\mathrm{Rh} 1>\mathrm{Ctrl}$ flies raised in either $\mathrm{LD}$ and DD conditions at D10 (Fig 5D-right). Thus, our data shows that Clock activity protects the Drosophila eye against oxidative stress. However, since expression of $\mathrm{Clk}^{\mathrm{DN}}$ only caused retinal degeneration in flies reared in LD, it is unlikely that the increased ROS levels are responsible for the retinal degeneration observed in $\mathrm{Clk}^{\mathrm{DN}}$ flies at D10.

Together, these data suggest that the disruption in expression of the phototransduction machinery in photoreceptors that lack Clock activity is likely responsible for the light-dependent retinal degeneration that we observed upon expression of $\mathrm{Clk}^{\mathrm{DN}}$. When we performed GO term analysis of genes that were differentially expressed in $\mathrm{Rh} 1>\mathrm{Clk}{ }^{\mathrm{DN}}$ at both $\mathrm{D} 10$ and D1, we observed significant upregulation of genes associated with response to unfolded protein and response to topologically incorrect protein, including many heat shock proteins and 
chaperones (Fig 5E and 5F). When the light-sensing Rhodopsin 1 (Rh1) is not properly inactivated, photoreceptor neurons experience substantial protein misfolding and ER stress, which leads to retinal degeneration in a light-dependent manner [55]. Since many of the genes that regulate Rhodopsin folding and inactivation were downregulated in $\mathrm{Rh} 1>\mathrm{Clk}^{\mathrm{DN}}$ relative to Rh1 >Ctrl flies, our data suggest that the circadian clock directly contributes to Rhodopsin maintenance in Drosophila photoreceptors. Importantly, the onset of light-dependent retinal degeneration associated with failures in Rh1 inactivation can be caused by several factors, including excessive endocytosis of $\mathrm{Rh} 1$ and increased $\mathrm{Ca}^{2+}$ influx [55,60-62]. We propose that Clock activity is protective in the retina because it promotes expression of genes that contribute to proper recycling of Rh1 upon light-exposure. However, our data also uncover an additional neuroprotective role of Clock by contributing to the response to oxidative stress, which might be important for additional tissues that do not contain the phototransduction machinery. This secondary role of Clock in the cellular response to oxidative stress might become increasingly important in advanced age, since accumulation of ROS is one of the hallmarks of aging.

\section{Discussion}

The circadian clock maintains daily biological rhythms by controlling the expression of target output genes, and is highly conserved between Drosophila and humans [41]. Since many genes are rhythmically expressed in the retina including most of the phototransduction machinery $[51,63,64]$, these observations suggest that the circadian clock plays a role in homeostatic regulation of the Drosophila retina. Here, we report that retina-specific expression of a dominant negative mutant of Clk in Drosophila leads to progressive light-dependent retinal degeneration and oxidative stress, showing that the circadian clock is required to maintain Drosophila photoreceptor integrity. Importantly, this role for the circadian clock in maintaining visual health is conserved in mammals because retina-specific disruption of BMAL1, the mice homolog of $c y c$, accelerates the loss of cone viability and function in aging $c h \times 10^{\mathrm{Cre}} ; \mathrm{Bmal1}{ }^{\mathrm{FlFl}}$ mice, which otherwise show a normal lifespan [65]. Further, disruption of BMAL1 leads to loss of spectral identity and integrity of cone cells in $\mathrm{Cr} x$-Cre; $B \mathrm{Bal1}^{\mathrm{Fl} / F l}[66]$, and the rat retina shows a circadian-dependent loss of resistance to light-mediated damage [67,68]. The circadian clock also plays a broader role in maintaining health during aging because mice deficient in BMAL1 in all tissues have reduced lifespan and several symptoms of premature aging including cataracts and neurodegeneration [69-71]. Moreover, homozygous $\mathrm{Per}^{\text {luc }}$ mice exhibit age-dependent photoreceptor degeneration and premature aging of the retinal pigment epithelium [72]. Since the PER::LUCIFERASE fusion protein is wild type and is used as a model for studying circadian rhythms [73], this observation suggests that even very mild changes in the expression or function of core circadian clock regulators can negatively impact the health of the aging eye. Notably, disruption of circadian rhythms in the human eye contributes to glaucoma and is also implicated in development of myopia $[74,75]$. We note that while preparing this manuscript, a preprint showed that expression of $\mathrm{Clk}^{\mathrm{DN}}$ under the photoreceptor-specific trpl-Gal4 driver caused decreased phototactic behavior in young flies relative to an age-matched control [76], which is consistent with the rhabdomere loss observed in our study. However, rhabdomere integrity was not tested by the authors of this study. Therefore, our studies suggest that the Drosophila retina serves as a useful model to study circadian-dependent regulation of photoreceptor homeostasis. It is important to note that Clk and Cyc could also have Clock-independent roles, as shown previously for aging brains [57]. Therefore, it will be important for future studies to establish Clock-dependent and -independent functions of Clk and Cyc in fly photoreceptors. 
How does disruption of the circadian clock in photoreceptors lead to light-dependent retinal degeneration? Our data suggest that although Clk-dependent transcription is necessary to prevent high levels of oxidative stress in the eye, this is not the proximal cause of retinal degeneration in young flies. Instead, we favor a model in which Clk:Cyc directly binds and activates expression of genes encoding the phototransduction machinery in photoreceptors, maintaining the continued expression of these proteins that have an integral role in photoreceptor structure and function. Numerous studies have demonstrated that complete loss of function of individual phototransduction genes results in retinal degeneration, often dependent on light exposure [55]. Our data show a significant and substantial decrease in transcript levels of multiple phototransduction genes, and we propose that the cumulative decrease in expression of their corresponding proteins causes the light-dependent retinal degeneration in flies expressing $\mathrm{Clk}^{\mathrm{DN}}$. However, because disrupting Clk:Cyc activity in photoreceptors had widespread effects on gene expression and chromatin accessibility, we cannot exclude the possibility that other pathways such as autophagy [77], $\mathrm{Ca}^{2+}$ signaling [78] and phosphoinositide metabolism [79] also contribute to the onset of light-dependent retinal degeneration.

The circadian clock has been implicated in the oxidative stress response in Drosophila $[57,80]$ and in mammals [81]. Since the onset of many age-related eye diseases is particularly sensitive to disruptions in oxidative stress response [82], an increase in ROS levels in $\mathrm{Rh} 1>\mathrm{Clk}^{\mathrm{DN}}$ eyes suggests an overall neuroprotective role for the circadian clock regulators in the retina, one which might become increasingly important with advanced age. Under standard laboratory conditions, wild-type flies with pigmented eyes only begin to exhibit the first signs of retinal degeneration after D50 [83]. Thus, we hypothesize that the increase in Clk:Cyc activity in aging photoreceptors protects against retinal degeneration in part, by promoting expression of genes that combat oxidative stress. Supporting this hypothesis, several stress response genes exhibit cyclic expression patterns in the head only in older flies (D55), and this cyclic expression is dependent on both Clk and oxidative stress [63]. However, because these studies were performed in female white-eyed $w^{1118}$ flies [63], which already show substantial retinal degeneration by $\mathrm{D} 15$ to $\mathrm{D} 30$ [84], it remains to be elucidated if these age-associated changes in cyclic transcription also occur in flies with pigmented eyes.

Here, we identified an age-dependent increase in Clk and Cyc TF activity in photoreceptors via an unbiased integrative ATAC-seq and RNA-seq approach, which focused on identifying changes in TF activity during aging based on changes in chromatin accessibility around TF binding sites. Thus, our initial approach was not focused on circadian biology and the data was obtained from single time-point comparisons; samples were collected at ZT6 $+/-1$ for aging experiments and at ZT9 $+/-1$ for the $\mathrm{Clk}^{\mathrm{DN}}$ experiments. Because of these sampling differences, the increase in Clk:Cyc TF activity in aging could reflect a change in the phase of Clk: Cyc binding to DNA during the day, as shown for Monarch butterfly brains [85], an increase in amplitude of binding activity, or both. Supporting the latter possibility, circadian analysis of the transcriptome of aging fly heads showed both an increase in the amplitude and a slight shift in phase of tim and per expression, moving earlier in the day as flies aged [63]. Other studies have observed a decrease in protein levels of the Clock repressor PER with age in Drosophila photoreceptors but not in pacemaker neurons [86,87], suggesting that the increased Clk: Cyc activity observed in our study could in fact reflect an increase in DNA binding of Clk:Cyc in old flies mediated by decreased repression by the Per:Tim complex. Nonetheless, the mechanisms underlying the age-associated changes in the circadian clock and Clk:Cyc TF activity in fly photoreceptors remain to be elucidated.

Overall, our work identifies a central role for the circadian clock in regulating the photoreceptor transcriptome. We observed that Clk:Cyc contributes to the expression of many thousands of genes in adult photoreceptors, consistent with reports from mammalian cells showing 
that Clock activity regulates the expression of $15 \%$ of expressed genes [53]. Clk and Cyc have been proposed to act at the top of a regulatory hierarchy to control widespread cyclic transcription in many cell types by regulating the expression of TFs [51,52]. Our data from photoreceptors are consistent with Clk:Cyc regulating expression of several important eye-specific TFs in Drosophila photoreceptors, suggesting a mechanism through which Clk and Cyc control expression of many genes indirectly. This is likely a widespread phenomenon across Drosophila tissues because gene regulatory network analysis of cyclic transcripts in brain, gut, Malphigian tubules, and fat bodies also identified many Clock-regulated TFs, including $h$, $h$ th, Mitf, and GATAd [88].

Interestingly, $\operatorname{per}\left(\mathrm{per}^{01}\right)$ and $\operatorname{tim}_{\left(\mathrm{tim}^{01}\right)}$ ) male mutants have extended lifespan, mediated by a loss of PER in intestine cells [89], suggesting that increased Clock activity in a particular tissue can lead to positive outcomes associated with health- and lifespan in a tissue- and sex-specific manner. Thus, identifying the molecular mechanism that underlie the age-associated changes in Clk:Cyc activity in aging photoreceptors and their impact on cellular homeostasis, which may be specific to the retina or other peripheral clocks in flies, will be an important area of research for future studies.

\section{Materials and methods}

\section{Fly collection and maintenance}

Rh1-Gal4 $>$ UAS-GFP ${ }^{\mathrm{KASH}}\left(w^{1118} ; ; P\left\{w^{+m C}=[U A S-G F P-M s p 300 K A S H\} a t t P 2, P\left\{r y^{+t 7.2}=\right.\right.\right.$ $r h 1-G A L 4\} 3, r y^{506}$ ) [3] flies were maintained on standard fly food as previously described. For aging experiments, flies were collected in a 3 day window after eclosion and transferred to population cages. For $\mathrm{Clk}^{\mathrm{DN}}$ experiments, flies were collected in a 24 hour period and transferred to population cages. D1 corresponds to flies that were collected the first day immediately after eclosion. Flies for both aging and $\mathrm{Clk}^{\mathrm{DN}}$ experiments were maintained in a $25^{\circ} \mathrm{C}$ incubator with a 12:12 hour light:dark cycle, where relative Zeitgeber Time (ZT) 0 corresponds to when the light cycle begins. For aging experiments, male flies were collected and flash-frozen at ZT6 $(-/+) 1$ hour. For Clk ${ }^{\mathrm{DN}}$ experiments, male flies were collected and flash-frozen at ZT9 $(-/+) 1$ hour. We note that UAS-GFP ${ }^{\mathrm{KASH}}$ and additional tagged KASH or QUAS flies [22] are available at Bloomington Drosophila Stock Center (BDSC) for nuclei immunoprecipitation in different tissues (\#92580 for the UAS-GFP ${ }^{\mathrm{KASH}}$ flies used in this study). Rh1-Gal4 (BDSC \#8691), $\mathrm{UAS}^{-C l k}{ }^{\mathrm{DN}}$ [\#1] (BDSC \#36319), UAS-Clk ${ }^{\mathrm{DN}}$ [\#2] (BDSC \#36318) and UAS-LacZ (BDSC \#8529) fly lines were obtained from BDSC. UAS-Cyc ${ }^{\mathrm{DN}}$ were generously provided by Dr. Daniel Cavanaugh (Loyola University)

\section{Chromatin accessibility and transcriptome analysis of photoreceptors}

Nuclei immuno-enrichment (NIE), Omni-ATAC, quantitative PCR (qPCR), and RNA-seq were performed on male Rh1-Gal4>UAS-GFP ${ }^{K A S H}$ flies at the indicated ages as previously described [22]. Briefly, for NIE experiments, we processed 400 age-matched male flies per replicate. Three independent biological replicates were obtained and analyzed for all RNA-seq and ATAC-seq samples. RNA-seq and ATAC-seq data analysis were performed as described in $[22,83]$; details specific to this study are described below. The following primers were used for qPCR: $\mathrm{Cl}^{D N}$ (5'- CGACAAGGATGATACAGATCAG-3' and 5'- ATTGCTGAGGAACG CAGGCT-3'); Clk $^{\text {WT }}$ (5'- GCGAGAAGAAGCGACGAGAT-3' and 5'- ATTGCTGAGGAAC GCAGGCT-3'); eIF1alpha (5'- GCTGGGCAACGGTCGTCTGGAGGC-3' and 5'- CGTCT TCAGGTTCCTGGCCTCGTCCGG-3'); RpL32 (5'- GCTAAGCTGTCGCACAAATG-3' and 5'- CGTTGTGCACCAGGAACTT-3'). 
Note, we used the same reverse primer to detect either WT or DN Clk transcript by combining with corresponding Forward primer (Fwd).

RNA-seq fold change heatmap analysis: Heatmaps were obtained using $\log _{2}$-transformed fold change values obtained from pair-wise comparisons using DESeq2 [90]. For aging heatmaps, DEGs obtained from each comparison with D10 were pooled and de-duplicated. Plots were obtained using R (v4.0.3) package pheatmap (v1.0.12).

Differential TF activity using diffTF: DiffTF [27] analysis was performed using the default parameters: analytical approach (nPermutations $=0)$, integration mode using raw counts obtained from STAR (-quantMode GeneCounts) [91], narrow peaks obtained using MACS2 [92]. TFBS bed files were obtained from PWMScan [93] using the Aug 2014 BDGP Rel6 + ISO1 MT/dm6 target genome with the 353 available motifs from the CIS-BP library [29] with default parameters ( $p$-value $<0.00001, \mathrm{Bg}$ base composition 0.29,0.21,0.21,0.29). Identified TFs were classified as significant if they had an FDR lower than 0.001 in at least one of the pair-wise aging comparisons with D10, or in either D1 or D10 analysis for the $\mathrm{Clk}^{\mathrm{DN}}$ comparisons. Detailed protocols for NIE, RNA-seq, Omni-ATAC, ChIP-seq, and CUT\&Tag are available at: dx.doi.org/10.17504/protocols.io.buiqnudw.

Clk ChIP-seq peak and photoreceptor ATAC-seq overlap: Previously published high-confidence peaks obtained from Clk and Cyc ChIP-seq data [51] were downloaded, and genomic coordinates were transformed from the $d m 3$ to $d m 6$ genome using the LiftOver tool from the UCSC Genome Browser website [94]. Genomic overlap was calculated using R (v4.0.3) packages ChIPpeakAnno (v3.24.2) and GenomicRanges (v.1.42.0).

Transcript per million (TPM) scatter plots: TPM values for each sample and replicate were obtained using TPMCalculator [95]. TPMs were averaged between biological replicates and used for scatter plot generation using R package ggplot2 (v3.3.3). DEGs obtained with DESeq2 were colored on the TPM scatter plots.

\section{Optic neutralization}

Optic neutralization and retinal degeneration quantification were performed as previously described $[17,96]$. Briefly, flies were glued to a glass slide using transparent nail-polish, and eyes imaged using bright-field microscopy. We note that optical neutralization using brightfield microscopy (with white light) is only possible with flies that have pigmented eyes, which is the case for all the flies tested in the present manuscript (See S5B Fig). Five biological replicates were analyzed for each sample, and retinal degeneration scores were assessed blindly.

\section{Targeted GSH:GSSG metabolomic assay}

25 eyes per sample were collected from male flies of the indicated age, condition, and genotype $(\mathrm{n}=3)$. Eyes were placed in a Covaris MicroTube with $110 \mu \mathrm{l}$ of blocking solution $(62.5 \mathrm{mg}$ $\mathrm{NEM}, 3 \mathrm{mg}$ EDTA, $5 \mathrm{mg} \mathrm{NaHO}$ disolved in $2 \mathrm{~mL}$ of 3:2 parts Water/MeOH (v/v)). Once all eyes were added to the blocking solution $10 \mu \mathrm{l}$ of $100 \mathrm{ng} / \mathrm{ul}$ of each internal standard was added ${ }^{13} \mathrm{C}_{2^{-}}{ }^{15} \mathrm{~N}-\mathrm{GSH}-\mathrm{NEM}$ and ${ }^{13} \mathrm{C}_{4^{-}}{ }^{15} \mathrm{~N}_{2}$-GSSG. Samples were homogenized using a Covaris Ultra Sonicator with the following settings: peak power: 200; duty factor: $10 \%$; cycles per burst: 200 ; time: 300 seconds. Samples were then processed using an Agilent 1260 Rapid Resolution liquid chromatography (LC) system coupled to an Agilent 6470 series QQQ mass spectrometer (MS/ MS) to analyze glutathione [97]. (Agilent Technologies, Santa Clara, CA). A Waters T3 2.1 $\mathrm{mm} \times 100 \mathrm{~mm}, 1.7 \mu \mathrm{m}$ column was used for LC separation (Water Corp, Milford, MA). The buffers were A) water $+0.1 \%$ formic acid and B) acetonitrile $+0.1 \%$ formic acid. The linear LC gradient was as follows: time 0 minutes, $0 \% \mathrm{~B}$; time 2 minutes, $0 \% \mathrm{~B}$; time 8 minutes, $30 \% \mathrm{~B}$; time 9 minutes, $95 \% \mathrm{~B}$; time 9.1 minutes, $0 \% \mathrm{~B}$; time 15 minutes, $0 \% \mathrm{~B}$. The flow rate was 0.3 
$\mathrm{mL} / \mathrm{min}$. Multiple reaction monitoring was used for MS analysis. Data were acquired in positive electrospray ionization (ESI) mode. The jet stream ESI interface had a gas temperature of $350^{\circ} \mathrm{C}$, gas flow rate of $9 \mathrm{~L} /$ minute, nebulizer pressure of $35 \mathrm{psi}$, sheath gas temperature of $300^{\circ} \mathrm{C}$, sheath gas flow rate of $9 \mathrm{~L} /$ minute, capillary voltage of $4000 \mathrm{~V}$ in positive mode, and nozzle voltage of $1000 \mathrm{~V}$. The $\Delta \mathrm{EMV}$ voltage was $450 \mathrm{~V}$. Agilent Masshunter Quantitative analysis software was used for data analysis (version 8.0).

\section{Supporting information}

S1 Fig. Pearson correlation aging RNA-seq. Pearson correlation heatmap of gene expression profiles from nuclear RNA-seq of aging samples $(n=3)$. Scores between 0 and 1 shown in each box correspond to Pearson's $r$ score.

(PDF)

S2 Fig. ATAC-seq peak genomic distribution. A. Bar plot showing the genomic distribution of accessible peaks identified in ATAC-seq data in Rh1 $>\mathrm{GFP}^{\mathrm{KASH}}$ during aging. Promoter annotation was based on $-/+2 \mathrm{~kb}$ around transcription start sites. B. DNA binding motif for $\mathrm{Clk}$ and Cyc derived from CIS-BP database. (PDF)

S3 Fig. DEG analysis in $\mathrm{Clk}^{\mathrm{DN}}$ photoreceptors. A. Bar plot showing $\log _{2}$-transformed transcript per million (TPM) values for core Clock genes comparing Rh1 $>\mathrm{Clk}^{\mathrm{DN}}$ and $\mathrm{Rh} 1>\mathrm{Ctrl}$ at either D1 (left) or D10 (right) (mean +- SD, n = 3, P-value obtained using t-test). B. Bar plot showing $C l k^{W T}$ and $C l k^{D N}$ transcript levels measured in heads from male flies of the indicated genotype. Transcript levels are normalized to the geometric mean of housekeeping genes eIF$1 \alpha$ and $R p L 32$ (mean $\pm \mathrm{SD} ; \mathrm{n}=3$, P-value obtained using $\mathrm{t}$-test). "ND" = not detected, “*” $=\mathrm{p}$ value $<0.05$, “**” $=$ p-value $<0.005$. C. Box plots showing the fold change values for genes identified as direct or indirect targets. Fold change is obtained using DESeq2. P-adjusted $>0.05$, | $\mathrm{FC} \mid>1$.5. P-value is obtained using Wilcoxon test. D,E. Complete ridge plot obtained from Gene Set Enrichment Analysis comparing differentially expressed genes in $\mathrm{Rh} 1>\mathrm{Clk}^{\mathrm{DN}}$ relative to Rh1 >Ctrl at D1 (D) or D10 (E). F. Volcano plot showing differentially expressed genes from photoreceptors when flies are reared in free-running conditions (dark:dark) versus light: dark cycle. Significance is equivalent to $-\log _{10}$ (adjusted p-value). Genes are colored based on changes in gene expression in D:D relative to L:D. Red is upregulated, and blue is downregulated. DEGs have p-adj < 0.05. G. Gene Ontology (GO) term analysis of genes that were significantly up-regulated (top) or down-regulated (bottom) in D:D relative to L:D.

(PDF)

S4 Fig. ATAC-seq analysis in $\mathrm{Clk}^{\mathrm{DN}}$ photoreceptors. A. Bar plot showing the genomic distribution of accessible peaks identified in ATAC-seq data from the indicated genotypes and ages. Promoter annotation was based on $-/+2 \mathrm{~kb}$ around transcription start sites. B. Volcano plots representing the differentially accessible peaks in $\mathrm{Rh} 1>\mathrm{Clk}^{\mathrm{DN}}$ relative to $\mathrm{Rh} 1>\mathrm{Ctrl}$. Differentially accessible peaks are defined as having a False Discovery Rate $(\mathrm{FDR})<0.05$, and absolute fold change $(|\mathrm{FC}|)>1.5$.

(PDF)

S5 Fig. Optic neutralization in Rh1 $>$ Cyc ${ }^{\text {DN }}$ flies. A. Heatmap showing RNA-seq fold change values for genes associated with phototransduction in flies expressing $\mathrm{Rh} 1>\mathrm{Clk}^{\mathrm{DN}}$ relative to Rh1 $>$ Ctrl at D1 or D10. Fold change values are represented inside each square, and colorlabelled based on fold change score. B. Images corresponding to representative flies from the indicated genotypes used to assess retinal degeneration via optical neutralization. Only males 
are shown, but females also showed similar eye pigmentation. C. Representative images of eyes from flies expressing $\mathrm{Cyc}^{\mathrm{DN}}$ at the indicated age reared in light:dark (LD) conditions. D. Clk and $c y c$ transcript ratios in aging samples. Kruskal-wallis test was used to determine differences amongst all groups, and pair-wise comparisons were obtained using standard $\mathrm{t}$-test. No comparison was significant.

(PDF)

S1 Table. DEGs identified during aging. Differentially expressed genes comparing each aging time point relative to $\mathrm{D} 10(\mathrm{n}=3)$. (XLSX)

S2 Table. DEGs identified in $\mathrm{Clk}^{\mathrm{DN}}$. Differentially expressed genes comparing $\mathrm{Rh} 1>\mathrm{Clk}^{\mathrm{DN}}$ relative to Control $(\mathrm{n}=3)$ at $\mathrm{D} 1$ or $\mathrm{D} 10$. (XLSX)

S3 Table. Gene list with Flybase IDs. List of genes used in the manuscript, with corresponding abbreviations, and Flybase gene IDs (XLSX)

S4 Table. Raw data retinal degeneration quantification. (XLSX)

S5 Table. Raw data redox glutathione measurements. (XLSX)

\section{Acknowledgments}

We thank the Weake lab for their comments on the manuscript. We thank Dr. Judith Zaugg (EMBL) and Dr. Paul Hardin (Texas A\&M) for reading and providing critical comments about the manuscript. We also thank Amber Jannasch for processing the metabolite samples. The authors acknowledge the use of the facilities of the Bindley Bioscience Center, a core facility of the NIH-funded Indiana Clinical and Translational Sciences Institute. We thank Dr.

Daniel Cavanaugh for providing the $\mathrm{Cyc}^{\mathrm{DN}}$ flies.

\section{Author Contributions}

Conceptualization: Juan Jauregui-Lozano.

Data curation: Juan Jauregui-Lozano.

Formal analysis: Juan Jauregui-Lozano.

Funding acquisition: Vikki M. Weake.

Investigation: Juan Jauregui-Lozano, Hana Hall, Sarah C. Stanhope, Kimaya Bakhle, Makayla M. Marlin.

Methodology: Juan Jauregui-Lozano.

Project administration: Vikki M. Weake.

Supervision: Vikki M. Weake.

Visualization: Juan Jauregui-Lozano.

Writing - original draft: Juan Jauregui-Lozano.

Writing - review \& editing: Juan Jauregui-Lozano, Vikki M. Weake. 


\section{References}

1. Bonnel S, Mohand-Said S, Sahel J-A. The aging of the retina. Experimental Gerontology. 2003; 38: 825-831. https://doi.org/10.1016/s0531-5565(03)00093-7 PMID: 12915204

2. Campello L, Singh N, Advani J, Mondal AK, Corso-Diaz X, Swaroop A. Aging of the Retina: Molecular and Metabolic Turbulences and Potential Interventions. Annu Rev Vis Sci. 2021. https://doi.org/10. 1146/annurev-vision-100419-114940 PMID: 34061570

3. Hall H, Medina P, Cooper DA, Escobedo SE, Rounds J, Brennan KJ, et al. Transcriptome profiling of aging Drosophila photoreceptors reveals gene expression trends that correlate with visual senescence. BMC Genomics. 2017; 18: 894. https://doi.org/10.1186/s12864-017-4304-3 PMID: 29162050

4. López-Otín C, Blasco MA, Partridge L, Serrano M, Kroemer G. The Hallmarks of Aging. Cell. 2013; 153: 1194-1217. https://doi.org/10.1016/j.cell.2013.05.039 PMID: 23746838

5. Parapuram SK, Cojocaru RI, Chang JR, Khanna R, Brooks M, Othman M, et al. Distinct signature of altered homeostasis in aging rod photoreceptors: implications for retinal diseases. PLoS One. 2010; 5: e13885. https://doi.org/10.1371/journal.pone.0013885 PMID: 21079736

6. Gemenetzi M, Lotery AJ. Epigenetics in age-related macular degeneration: new discoveries and future perspectives. Cell Mol Life Sci. 2020; 77: 807-818. https://doi.org/10.1007/s00018-019-03421-w PMID: 31897542

7. Li X, He S, Zhao M. An Updated Review of the Epigenetic Mechanism Underlying the Pathogenesis of Age-related Macular Degeneration. Aging Dis. 2020; 11: 1219-1234. https://doi.org/10.14336/AD 2019.1126 PMID: 33014534

8. Martins R, Lithgow GJ, Link W. Long live FOXO: unraveling the role of FOXO proteins in aging and longevity. Aging Cell. 2016; 15: 196-207. https://doi.org/10.1111/acel.12427 PMID: 26643314

9. Murtaza G, Khan AK, Rashid R, Muneer S, Hasan SMF, Chen J. FOXO Transcriptional Factors and Long-Term Living. Oxid Med Cell Longev. 2017; 2017. https://doi.org/10.1155/2017/3494289 PMID: 28894507

10. Sun X, Chen W-D, Wang Y-D. DAF-16/FOXO Transcription Factor in Aging and Longevity. Front Pharmacol. 2017; 8. https://doi.org/10.3389/fphar.2017.00548 PMID: 28878670

11. Bou Sleiman M, Jha P, Houtkooper R, Williams RW, Wang X, Auwerx J. The Gene-Regulatory Footprint of Aging Highlights Conserved Central Regulators. Cell Reports. 2020; 32: 108203. https://doi.org/ 10.1016/j.celrep.2020.108203 PMID: 32997995

12. Yan F, Powell DR, Curtis DJ, Wong NC. From reads to insight: a hitchhiker's guide to ATAC-seq data analysis. Genome Biol. 2020; 21: 22. https://doi.org/10.1186/s13059-020-1929-3 PMID: 32014034

13. Davie K, Jacobs J, Atkins M, Potier D, Christiaens V, Halder G, et al. Discovery of Transcription Factors and Regulatory Regions Driving In Vivo Tumor Development by ATAC-seq and FAIRE-seq Open Chromatin Profiling. PLoS Genet. 2015; 11. https://doi.org/10.1371/journal.pgen.1004994 PMID: 25679813

14. Buono L, Martinez-Morales J-R. Retina Development in Vertebrates: Systems Biology Approaches to Understanding Genetic Programs: On the Contribution of Next-Generation Sequencing Methods to the Characterization of the Regulatory Networks Controlling Vertebrate Eye Development. Bioessays. 2020; 42: e1900187. https://doi.org/10.1002/bies.201900187 PMID: 31997389

15. Potier D, Davie K, Hulselmans G, Naval Sanchez M, Haagen L, Huynh-Thu VA, et al. Mapping gene regulatory networks in Drosophila eye development by large-scale transcriptome perturbations and motif inference. Cell Rep. 2014; 9: 2290-2303. https://doi.org/10.1016/j.celrep.2014.11.038 PMID: 25533349

16. Wang J, Zibetti C, Shang P, Sripathi SR, Zhang P, Cano M, et al. ATAC-Seq analysis reveals a widespread decrease of chromatin accessibility in age-related macular degeneration. Nat Commun. 2018; 9. https://doi.org/10.1038/s41467-018-03856-y PMID: 29636475

17. Stegeman R, Hall H, Escobedo SE, Chang HC, Weake VM. Proper splicing contributes to visual function in the aging Drosophila eye. Aging Cell. 2018; 17. https://doi.org/10.1111/acel.12817 PMID: 30003673

18. Rister J, Desplan C. The retinal mosaics of opsin expression in invertebrates and vertebrates. Developmental Neurobiology. 2011; 71: 1212-1226. https://doi.org/10.1002/dneu.20905 PMID: 21557510

19. Montell C. Drosophila visual transduction. Trends in Neurosciences. 2012; 35: 356-363. https://doi.org/ 10.1016/j.tins.2012.03.004 PMID: 22498302

20. Piper MDW, Partridge L. Drosophila as a model for ageing. Biochimica et Biophysica Acta (BBA)— Molecular Basis of Disease. 2018; 1864: 2707-2717. https://doi.org/10.1016/j.bbadis.2017.09.016 PMID: 28964875 
21. Ma J, Weake VM. Affinity-based Isolation of Tagged Nuclei from Drosophila Tissues for Gene Expression Analysis. JoVE (Journal of Visualized Experiments). 2014; e51418. https://doi.org/10.3791/51418 PMID: 24686501

22. Jauregui-Lozano J, Bakhle K, Weake VM. In vivo tissue-specific chromatin profiling in Drosophila melanogaster using GFP-tagged nuclei. Genetics. 2021 [cited 22 May 2021]. https://doi.org/10.1093/ genetics/iyab079 PMID: 34022041

23. Grandison RC, Wong R, Bass TM, Partridge L, Piper MDW. Effect of a Standardised Dietary Restriction Protocol on Multiple Laboratory Strains of Drosophila melanogaster. PLOS ONE. 2009; 4: e4067. https://doi.org/10.1371/journal.pone.0004067 PMID: 19119322

24. Francois $\mathrm{M}$, Donovan $\mathrm{P}$, Fontaine $\mathrm{F}$. Modulating transcription factor activity: Interfering with protein-protein interaction networks. Seminars in Cell \& Developmental Biology. 2020; 99: 12-19. https://doi.org/ 10.1016/j.semcdb.2018.07.019 PMID: 30172762

25. Filtz TM, Vogel WK, Leid M. Regulation of transcription factor activity by interconnected, post-translational modifications. Trends Pharmacol Sci. 2014; 35: 76-85. https://doi.org/10.1016/j.tips.2013.11.005 PMID: 24388790

26. Hall H, Cooper BR, Qi G, Wijeratne AB, Mosley AL, Weake VM. Quantitative Proteomic and Metabolomic Profiling Reveals Altered Mitochondrial Metabolism and Folate Biosynthesis Pathways in the Aging Drosophila Eye. Molecular \& Cellular Proteomics. 2021; 20: 100127. https://doi.org/10.1016/j.mcpro. 2021.100127 PMID: 34332122

27. Berest I, Arnold C, Reyes-Palomares A, Palla G, Rasmussen KD, Giles H, et al. Quantification of Differential Transcription Factor Activity and Multiomics-Based Classification into Activators and Repressors: diffTF. Cell Reports. 2019; 29: 3147-3159.e12. https://doi.org/10.1016/j.celrep.2019.10.106 PMID: 31801079

28. Hammelman J, Patel T, Closser M, Wichterle H, Gifford D. Ranking Reprogramming Factors for Directed Differentiation. bioRxiv. 2021; 2021.05.14.444080. https://doi.org/10.1101/2021.05.14.444080

29. Weirauch MT, Yang A, Albu M, Cote AG, Montenegro-Montero A, Drewe P, et al. Determination and inference of eukaryotic transcription factor sequence specificity. Cell. 2014; 158: 1431-1443. https:// doi.org/10.1016/j.cell.2014.08.009 PMID: 25215497

30. Heinz S, Benner C, Spann N, Bertolino E, Lin YC, Laslo P, et al. Simple combinations of lineage-determining transcription factors prime cis-regulatory elements required for macrophage and $B$ cell identities. Mol Cell. 2010; 38: 576-589. https://doi.org/10.1016/j.molcel.2010.05.004 PMID: 20513432

31. Ramírez F, Bhardwaj V, Arrigoni L, Lam KC, Grüning BA, Villaveces J, et al. High-resolution TADs reveal DNA sequences underlying genome organization in flies. Nat Commun. 2018; 9: 189. https://doi. org/10.1038/s41467-017-02525-w PMID: 29335486

32. Vinayagam A, Kulkarni MM, Sopko R, Sun X, Hu Y, Nand A, et al. An Integrative Analysis of the InR/ PI3K/Akt Network Identifies the Dynamic Response to Insulin Signaling. Cell Reports. 2016; 16: 30623074. https://doi.org/10.1016/j.celrep.2016.08.029 PMID: 27626673

33. Shokri L, Inukai S, Hafner A, Weinand K, Hens K, Vedenko A, et al. A Comprehensive Drosophila melanogaster Transcription Factor Interactome. Cell Reports. 2019; 27: 955-970.e7. https://doi.org/10. 1016/j.celrep.2019.03.071 PMID: 30995488

34. Haigis MC, Yankner BA. The Aging Stress Response. Mol Cell. 2010; 40: 333-344. https://doi.org/10. 1016/j.molcel.2010.10.002 PMID: 20965426

35. Zhang K, Chaillet JR, Perkins LA, Halazonetis TD, Perrimon N. Drosophila homolog of the mammalian jun oncogene is expressed during embryonic development and activates transcription in mammalian cells. Proc Natl Acad Sci U S A. 1990; 87: 6281-6285. https://doi.org/10.1073/pnas.87.16.6281 PMID: 1696724

36. Dekanty A, Lavista-Llanos S, Irisarri M, Oldham S, Wappner $P$. The insulin-PI3K/TOR pathway induces a HIF-dependent transcriptional response in Drosophila by promoting nuclear localization of HIF-a/ Sima. Journal of Cell Science. 2005; 118: 5431-5441. https://doi.org/10.1242/jcs.02648 PMID: 16278294

37. Baird NA, Turnbull DW, Johnson EA. Induction of the Heat Shock Pathway during Hypoxia Requires Regulation of Heat Shock Factor by Hypoxia-inducible Factor-1*. Journal of Biological Chemistry. 2006; 281: 38675-38681. https://doi.org/10.1074/jbc.M608013200 PMID: 17040902

38. Chakrabarti S, Poidevin M, Lemaitre B. The Drosophila MAPK p38c Regulates Oxidative Stress and Lipid Homeostasis in the Intestine. PLOS Genetics. 2014; 10: e1004659. https://doi.org/10.1371/ journal.pgen.1004659 PMID: 25254641

39. Sano Y, Akimaru H, Okamura T, Nagao T, Okada M, Ishii S. Drosophila Activating Transcription Factor2 Is Involved in Stress Response via Activation by p38, but Not c-Jun NH2-Terminal Kinase. MBoC. 2005; 16: 2934-2946. https://doi.org/10.1091/mbc.e04-11-1008 PMID: 15788564 
40. Hall BS, Barnett YA, Crofts JJ, Chuzhanova N. Identification of novel genes associated with longevity in Drosophila melanogaster-a computational approach. Aging (Albany NY). 2019; 11: 11244-11267. https://doi.org/10.18632/aging.102527 PMID: 31794428

41. Patke A, Young MW, Axelrod S. Molecular mechanisms and physiological importance of circadian rhythms. Nat Rev Mol Cell Biol. 2020; 21: 67-84. https://doi.org/10.1038/s41580-019-0179-2 PMID: 31768006

42. Menet JS, Hardin PE. Circadian Clocks: The tissue is the issue. Curr Biol. 2014; 24: R25-R27. https:// doi.org/10.1016/j.cub.2013.11.016 PMID: 24405673

43. Giebultowicz JM, Long DM. Ageing and Circadian rhythms. Curr Opin Insect Sci. 2015; 7: 82-86. https://doi.org/10.1016/j.cois.2015.03.001 PMID: 26000238

44. Hood S, Amir S. The aging clock: circadian rhythms and later life. J Clin Invest. 2017; 127: 437-446. https://doi.org/10.1172/JCl90328 PMID: 28145903

45. Trott AJ, Menet JS. Regulation of circadian clock transcriptional output by CLOCK:BMAL1. PLoS Genet. 2018; 14: e1007156. https://doi.org/10.1371/journal.pgen.1007156 PMID: 29300726

46. Hardin PE. Transcription Regulation within the Circadian Clock: The E-box and Beyond. J Biol Rhythms. 2004; 19: 348-360. https://doi.org/10.1177/0748730404268052 PMID: 15534316

47. Tanoue S, Krishnan P, Krishnan B, Dryer SE, Hardin PE. Circadian Clocks in Antennal Neurons Are Necessary and Sufficient for Olfaction Rhythms in Drosophila. Current Biology. 2004; 14: 638-649. https://doi.org/10.1016/j.cub.2004.04.009 PMID: 15084278

48. Mahesh G, Rivas GBS, Caster C, Ost EB, Amunugama R, Jones R, et al. Proteomic analysis of Drosophila CLOCK complexes identifies rhythmic interactions with SAGA and Tip60 complex component NIPPED-A. Sci Rep. 2020; 10. https://doi.org/10.1038/s41598-020-75009-5 PMID: 33087840

49. Richier B, Michard-Vanhée C, Lamouroux A, Papin C, Rouyer F. The clockwork orange Drosophila protein functions as both an activator and a repressor of clock gene expression. J Biol Rhythms. 2008; 23: 103-116. https://doi.org/10.1177/0748730407313817 PMID: 18375860

50. Mollereau B, Wernet MF, Beaufils P, Killian D, Pichaud F, Kühnlein R, et al. A green fluorescent protein enhancer trap screen in Drosophila photoreceptor cells. Mechanisms of Development. 2000; 93: 151160. https://doi.org/10.1016/s0925-4773(00)00287-2 PMID: 10781948

51. Meireles-Filho ACA, Bardet AF, Yáñez-Cuna JO, Stampfel G, Stark A. cis-regulatory requirements for tissue-specific programs of the circadian clock. Curr Biol. 2014; 24: 1-10. https://doi.org/10.1016/j.cub. 2013.11.017 PMID: 24332542

52. Abruzzi KC, Rodriguez J, Menet JS, Desrochers J, Zadina A, Luo W, et al. Drosophila CLOCK target gene characterization: implications for circadian tissue-specific gene expression. Genes Dev. 2011; 25: 2374-2386. https://doi.org/10.1101/gad.178079.111 PMID: 22085964

53. Menet JS, Pescatore S, Rosbash M. CLOCK:BMAL1 is a pioneer-like transcription factor. Genes Dev. 2014; 28: 8-13. https://doi.org/10.1101/gad.228536.113 PMID: 24395244

54. Glossop NRJ, Houl JH, Zheng H, Ng FS, Dudek SM, Hardin PE. VRILLE Feeds Back to Control Circadian Transcription of Clock in the Drosophila Circadian Oscillator. Neuron. 2003; 37: 249-261. https:// doi.org/10.1016/s0896-6273(03)00002-3 PMID: 12546820

55. Wang T, Montell C. Phototransduction and retinal degeneration in Drosophila. Pflugers Arch-Eur J Physiol. 2007; 454: 821-847. https://doi.org/10.1007/s00424-007-0251-1 PMID: 17487503

56. Allada R, Kadener S, Nandakumar N, Rosbash M. A recessive mutant of Drosophila Clock reveals a role in circadian rhythm amplitude. EMBO J. 2003; 22: 3367-3375. https://doi.org/10.1093/emboj/ cdg318 PMID: 12839998

57. Vaccaro A, Issa A-R, Seugnet L, Birman S, Klarsfeld A. Drosophila Clock Is Required in Brain Pacemaker Neurons to Prevent Premature Locomotor Aging Independently of Its Circadian Function. PLOS Genetics. 2017; 13: e1006507. https://doi.org/10.1371/journal.pgen.1006507 PMID: 28072817

58. Domènech E B., Marfany G. The Relevance of Oxidative Stress in the Pathogenesis and Therapy of Retinal Dystrophies. Antioxidants (Basel). 2020; 9. https://doi.org/10.3390/antiox9040347 PMID: 32340220

59. ZITKA O, SKALICKOVA S, GUMULEC J, MASARIK M, ADAM V, HUBALEK J, et al. Redox status expressed as GSH:GSSG ratio as a marker for oxidative stress in paediatric tumour patients. Oncol Lett. 2012; 4: 1247-1253. https://doi.org/10.3892/ol.2012.931 PMID: 23205122

60. Gorbatyuk M, Gorbatyuk O. Review: Retinal degeneration: Focus on the unfolded protein response. Mol Vis. 2013; 19: 1985-xxx. PMID: 24068865

61. Shinde V, Kotla P, Strang C, Gorbatyuk M. Unfolded protein response-induced dysregulation of calcium homeostasis promotes retinal degeneration in rat models of autosomal dominant retinitis pigmentosa. Cell Death Dis. 2016; 7: e2085-e2085. https://doi.org/10.1038/cddis.2015.325 PMID: 26844699 
62. Geng C, Pak WL. Photoreceptor Degeneration and Ca2+ Influx through Light-Activated Channels of Drosophila. Madame Curie Bioscience Database [Internet]. Landes Bioscience; 2013. Available: https://www.ncbi.nlm.nih.gov/books/NBK6284/

63. Kuintzle RC, Chow ES, Westby TN, Gvakharia BO, Giebultowicz JM, Hendrix DA. Circadian deep sequencing reveals stress-response genes that adopt robust rhythmic expression during aging. Nature Communications. 2017; 8: 14529. https://doi.org/10.1038/ncomms14529 PMID: 28221375

64. Damulewicz M, Świątek M, Łoboda A, Dulak J, Bilska B, Przewłocki R, et al. Daily Regulation of Phototransduction, Circadian Clock, DNA Repair, and Immune Gene Expression by Heme Oxygenase in the Retina of Drosophila. Genes (Basel). 2018; 10: 6. https://doi.org/10.3390/genes10010006 PMID: 30583479

65. Baba K, Piano I, Lyuboslavsky P, Chrenek MA, Sellers JT, Zhang S, et al. Removal of clock gene Bmal1 from the retina affects retinal development and accelerates cone photoreceptor degeneration during aging. PNAS. 2018; 115: 13099-13104. https://doi.org/10.1073/pnas.1808137115 PMID: 30498030

66. Sawant OB, Horton AM, Zucaro OF, Chan R, Bonilha VL, Samuels IS, et al. The Circadian Clock Gene Bmal1 Controls Thyroid Hormone-Mediated Spectral Identity and Cone Photoreceptor Function. Cell Rep. 2017; 21: 692-706. https://doi.org/10.1016/j.celrep.2017.09.069 PMID: 29045837

67. Grewal R, Organisciak D, Wong P. Factors underlying circadian dependent susceptibility to light induced retinal damage. Adv Exp Med Biol. 2006; 572: 411-416. https://doi.org/10.1007/0-387-324429 58 PMID: 17249604

68. Organisciak DT, Darrow RM, Barsalou L, Kutty RK, Wiggert B. Circadian-Dependent Retinal Light Damage in Rats. Investigative Ophthalmology \& Visual Science. 2000; 41: 3694-3701. PMID: 11053264

69. Kondratov RV, Kondratova AA, Gorbacheva VY, Vykhovanets OV, Antoch MP. Early aging and agerelated pathologies in mice deficient in BMAL1, the core componentof the circadian clock. Genes Dev. 2006; 20: 1868-1873. https://doi.org/10.1101/gad.1432206 PMID: 16847346

70. Aging | Premature aging of the hippocampal neurogenic niche in adult Bmal1- deficient mice-Full Text. [cited 26 Apr 2021]. Available: https://www.aging-us.com/article/100764/text

71. Musiek ES, Lim MM, Yang G, Bauer AQ, Qi L, Lee Y, et al. Circadian clock proteins regulate neuronal redox homeostasis and neurodegeneration. J Clin Invest. 2013; 123: 5389-5400. https://doi.org/10. 1172/JCI70317 PMID: 24270424

72. Goyal V, DeVera C, Baba K, Sellers J, Chrenek MA, luvone PM, et al. Photoreceptor Degeneration in Homozygous Male Per2luc Mice During Aging. J Biol Rhythms. 2020; 0748730420965285. https://doi. org/10.1177/0748730420965285 PMID: 33135952

73. Yoo S-H, Yamazaki S, Lowrey PL, Shimomura K, Ko CH, Buhr ED, et al. PERIOD2::LUCIFERASE real-time reporting of circadian dynamics reveals persistent circadian oscillations in mouse peripheral tissues. Proc Natl Acad Sci U S A. 2004; 101: 5339-5346. https://doi.org/10.1073/pnas.0308709101 PMID: 14963227

74. Martínez-Águila A, Martín-Gil A, Carpena-Torres C, Pastrana C, Carracedo G. Influence of Circadian Rhythm in the Eye: Significance of Melatonin in Glaucoma. Biomolecules. 2021; 11. https://doi.org/10 3390/biom11030340 PMID: 33668357

75. Ko GY-P. Circadian regulation in the retina: From molecules to network. Eur J Neurosci. 2020; 51: 194216. https://doi.org/10.1111/ejn.14185 PMID: 30270466

76. Hodge BA, Meyerhof GT, Katewa SD, Lian T, Lau C, Bar S, et al. Dietary restriction and clock delay eye aging to extend lifespan in D. melanogaster. 2021 May p. 2021.05.08.443272. https://doi.org/10.1101/ 2021.05.08.443272

77. Ma D, Li S, Molusky MM, Lin JD. Circadian autophagy rhythm: a link between clock and metabolism? Trends Endocrinol Metab. 2012; 23: 319-325. https://doi.org/10.1016/j.tem.2012.03.004 PMID: 22520961

78. Cavieres-Lepe J, Ewer J. Reciprocal Relationship Between Calcium Signaling and Circadian Clocks: Implications for Calcium Homeostasis, Clock Function, and Therapeutics. Frontiers in Molecular Neuroscience. 2021; 14: 83. https://doi.org/10.3389/fnmol.2021.666673 PMID: 34045944

79. Mustafi D, Kevany BM, Genoud C, Bai X, Palczewski K. Photoreceptor phagocytosis is mediated by phosphoinositide signaling. FASEB J. 2013; 27: 4585-4595. https://doi.org/10.1096/fj.13-237537 PMID: 23913857

80. Krishnan N, Davis AJ, Giebultowicz JM. Circadian regulation of response to oxidative stress in Drosophila melanogaster. Biochemical and Biophysical Research Communications. 2008; 374: 299-303. https://doi.org/10.1016/j.bbrc.2008.07.011 PMID: 18627767 
81. Wilking M, Ndiaye M, Mukhtar H, Ahmad N. Circadian Rhythm Connections to Oxidative Stress: Implications for Human Health. Antioxid Redox Signal. 2013; 19: 192-208. https://doi.org/10.1089/ars.2012. 4889 PMID: 23198849

82. Rohowetz LJ, Kraus JG, Koulen P. Reactive Oxygen Species-Mediated Damage of Retinal Neurons: Drug Development Targets for Therapies of Chronic Neurodegeneration of the Retina. Int J Mol Sci. 2018; 19: 3362. https://doi.org/10.3390/ijms19113362 PMID: 30373222

83. Jauregui-Lozano J, Escobedo S, Easton A, Lanman NA, Weake VM, Hall H. Proper control of R-loop homeostasis is required for maintenance of gene expression and neuronal function during aging. Aging Cell; 2022; https://doi.org/10.1111/acel.13554 PMID: 35048512

84. Ferreiro MJ, Pérez C, Marchesano M, Ruiz S, Caputi A, Aguilera P, et al. Drosophila melanogaster White Mutant w1118 Undergo Retinal Degeneration. Frontiers in Neuroscience. 2018; 11: 732. https:// doi.org/10.3389/fnins.2017.00732 PMID: 29354028

85. Lugena AB, Zhang $Y$, Menet JS, Merlin C. Genome-wide discovery of the daily transcriptome, DNA regulatory elements and transcription factor occupancy in the monarch butterfly brain. PLoS Genet. 2019;15. https://doi.org/10.1371/journal.pgen.1008265 PMID: 31335862

86. Luo W, Chen W-F, Yue Z, Chen D, Sowcik M, Sehgal A, et al. Old flies have a robust central oscillator but weaker behavioral rhythms that can be improved by genetic and environmental manipulations. Aging Cell. 2012; 11: 428-438. https://doi.org/10.1111/j.1474-9726.2012.00800.x PMID: 22268765

87. Rakshit K, Krishnan N, Guzik EM, Elżbieta Pyza, Giebultowicz JM. Effects of Aging on the Molecular Circadian Oscillations in Drosophila. Chronobiology International. 2012; 29: 5-14. https://doi.org/10. 3109/07420528.2011.635237 PMID: 22217096

88. Litovchenko M, Meireles-Filho ACA, Frochaux MV, Bevers RPJ, Prunotto A, Anduaga AM, et al. Extensive tissue-specific expression variation and novel regulators underlying circadian behavior. Sci Adv. 2021; 7: eabc3781. https://doi.org/10.1126/sciadv.abc3781 PMID: 33514540

89. Ulgherait M, Chen A, McAllister SF, Kim HX, Delventhal R, Wayne CR, et al. Circadian regulation of mitochondrial uncoupling and lifespan. Nat Commun. 2020; 11: 1927. https://doi.org/10.1038/s41467020-15617-x PMID: 32317636

90. Love MI, Huber W, Anders S. Moderated estimation of fold change and dispersion for RNA-seq data with DESeq2. Genome Biol. 2014; 15: 550. https://doi.org/10.1186/s13059-014-0550-8 PMID: 25516281

91. Dobin A, Davis CA, Schlesinger F, Drenkow J, Zaleski C, Jha S, et al. STAR: ultrafast universal RNAseq aligner. Bioinformatics. 2013; 29: 15-21. https://doi.org/10.1093/bioinformatics/bts635 PMID: 23104886

92. Zhang Y, Liu T, Meyer CA, Eeckhoute J, Johnson DS, Bernstein BE, et al. Model-based analysis of ChIP-Seq (MACS). Genome Biol. 2008; 9: R137. https://doi.org/10.1186/gb-2008-9-9-r137 PMID: 18798982

93. Ambrosini G, Groux R, Bucher P. PWMScan: a fast tool for scanning entire genomes with a positionspecific weight matrix. Bioinformatics. 2018; 34: 2483-2484. https://doi.org/10.1093/bioinformatics/ bty127 PMID: 29514181

94. Kent WJ, Sugnet CW, Furey TS, Roskin KM, Pringle TH, Zahler AM, et al. The Human Genome Browser at UCSC. Genome Res. 2002; 12: 996-1006. https://doi.org/10.1101/gr.229102 PMID: 12045153

95. Vera Alvarez R, Pongor LS, Mariño-Ramírez L, Landsman D. TPMCalculator: one-step software to quantify mRNA abundance of genomic features. Bioinformatics. 2019; 35: 1960-1962. https://doi.org/ 10.1093/bioinformatics/bty896 PMID: 30379987

96. Chen X, Hall H, Simpson JP, Leon-Salas WD, Ready DF, Weake VM. Cytochrome b5 protects photoreceptors from light stress-induced lipid peroxidation and retinal degeneration. NPJ Aging Mech Dis. 2017; 3: 18. https://doi.org/10.1038/s41514-017-0019-6 PMID: 29214051

97. Florholmen-Kjær Å, Lyså RA, Fuskevåg O-M, Goll R, Revhaug A, Mortensen KE. A sensitive method for the analysis of glutathione in porcine hepatocytes. Scand J Gastroenterol. 2014; 49: 1359-1366. https://doi.org/10.3109/00365521.2014.964757 PMID: 25279941 